\title{
Translocation of a parthenogenesis gene candidate to an alternate carrier chromosome in apomictic Brachiaria humidicola
}

Margaret Worthington ${ }^{1,5^{*}}$ (D, Masumi Ebina ${ }^{2}$, Naoki Yamanaka ${ }^{3}$, Christopher Heffelfinger $^{4}$, Constanza Quintero $^{1}$, Yeny Patricia Zapata ${ }^{1}$, Juan Guillermo Perez ${ }^{1}$, Michael Selvaraj ${ }^{1}$, Manabu Ishitani ${ }^{1}$, Jorge Duitama ${ }^{1,6}$, Juan Fernando de la Hoz ${ }^{1,7}$, Idupulapati Rao ${ }^{1,8}$, Stephen Dellaporta ${ }^{4}$, Joe Tohme ${ }^{1}$ and Jacobo Arango ${ }^{1}$

\begin{abstract}
Background: The apomictic reproductive mode of Brachiaria (syn. Urochloa) forage species allows breeders to faithfully propagate heterozygous genotypes through seed over multiple generations. In Brachiaria, reproductive mode segregates as single dominant locus, the apospory-specific genomic region (ASGR). The AGSR has been mapped to an area of reduced recombination on Brachiaria decumbens chromosome 5. A primer pair designed within ASGR-BABY BOOM-like $(B B M L)$, the candidate gene for the parthenogenesis component of apomixis in Pennisetum squamulatum, was diagnostic for reproductive mode in the closely related species B. ruziziensis, B. brizantha, and B. decumbens. In this study, we used a mapping population of the distantly related commercial species $B$. humidicola to map the ASGR and test for conservation of ASGR-BBML sequences across Brachiaria species.
\end{abstract}

Results: Dense genetic maps were constructed for the maternal and paternal genomes of a hexaploid $(2 n=6 x=36) B$. humidicola $F_{1}$ mapping population ( $\left.n=102\right)$ using genotyping-by-sequencing, simple sequence repeat, amplified fragment length polymorphism, and transcriptome derived single nucleotide polymorphism markers. Comparative genomics with Setaria italica provided confirmation for $x=6$ as the base chromosome number of $B$. humidicola. High resolution molecular karyotyping indicated that the six homologous chromosomes of the sexual female parent paired at random, whereas preferential pairing of subgenomes was observed in the apomictic male parent. Furthermore, evidence for compensated aneuploidy was found in the apomictic parent, with only five homologous linkage groups identified for chromosome 5 and seven homologous linkage groups of chromosome 6 . The ASGR mapped to B. humidicola chromosome 1, a region syntenic with chromosomes 1 and 7 of S. italica. The ASGR-BBML specific PCR product cosegregated with the ASGR in the $F_{1}$ mapping population, despite its location on a different carrier chromosome than B. decumbens.

Conclusions: The first dense molecular maps of $B$. humidicola provide strong support for cytogenetic evidence indicating a base chromosome number of six in this species. Furthermore, these results show conservation of the ASGR across the Paniceae in different chromosomal backgrounds and support postulation of the ASGR-BBML as candidate genes for the parthenogenesis component of apomixis.

Keywords: Apomixis, Apospory, Parthenogenesis, BABY BOOM, Aneuploidy, Segmental allopolyploidy, Genotyping-by-sequencing, Molecular karyotyping, Urochloa, Brachiaria

\footnotetext{
*Correspondence: mlworthi@uark.edu

1 International Center for Tropical Agriculture (CIAT), A.A. 6713, Cali, Colombia

${ }^{5}$ Present address: Department of Horticulture, University of Arkansas, 306

Plant Sciences Bldg, Fayetteville, AR 72701, USA

Full list of author information is available at the end of the article
}

(c) The Author(s). 2019 Open Access This article is distributed under the terms of the Creative Commons Attribution 4.0 International License (http://creativecommons.org/licenses/by/4.0/), which permits unrestricted use, distribution, and reproduction in any medium, provided you give appropriate credit to the original author(s) and the source, provide a link to the Creative Commons license, and indicate if changes were made. The Creative Commons Public Domain Dedication waiver (http://creativecommons.org/publicdomain/zero/1.0/) applies to the data made available in this article, unless otherwise stated. 


\section{Background}

Several important forage grass genera, including Brachiaria (Trin.) Griseb. (syn. Urochloa P. Beauv.), Cenchrus L./Pennisetum Rich., Panicum L. (syn. Megathrysus), and Paspalum L., reproduce via apomixis. Apomixis, asexual reproduction through seed, is a naturally occurring reproductive mode that enables breeders to select and faithfully propagate outstanding heterozygous genotypes without vegetative propagation or hybrid seed production. In apospory, the apomictic pathway found in Brachiaria and other Paniceae grass genera, unreduced embryo sacs first develop from an adjacent somatic nucellar cell in a process termed apomeiosis [1]. This unreduced embryo sac then develops into a viable embryo without fertilization through parthenogenesis [2, 3]. Pseudogamous fertilization of the secondary nuclei of apomictic embryo-sacs by viable pollen gametes is required for normal endosperm development to occur [3].

The apomeiosis and parthenogenesis components of apomixis are usually inherited together as a single dominant Mendelian factor denoted as the "apospory-specific genomic region" (ASGR) in Paniceae grasses [1]. The ASGR was mapped to a physically large hemizygous region of reduced recombination in Pennisetum squamulatum (L.) R. Br. (syn. Cenchrus squamulatus) [4], and Paspalum notatum Flüggé and Paspalum simplex Morong [5-9]. The ASGR-BABY BOOM-like (ASGR-BBML) genes have been posited as candidate genes for parthenogenesis in Cenchrus/Pennisetum [10]. The ASGR$B B M L$ have high sequence similarity to the $B A B Y$ $B O O M(B B M)$ genes associated with somatic embryogenesis in Brassica and Arabidopsis [11]. Furthermore, apomictic $F_{1}$ RNAi transgenic plants with reduced PSASGR-BBML expression had reduced parthenogenic embryo development and transgenic sexual pearl millet plants expressing $P$. squamulatum PSASGR-BBML genes were induced to produce haploid offspring [10].

A recent study in the genus Brachiaria provided additional support for the postulation of the ASGR-BBML as candidate genes for the apomictic function of parthenogenesis [12]. The psASGR-BBML-specific primer pair p779/p780 was developed from sequences in the 4th and 7th exons of P. squamulatum ASGR-BBM-like2 [13]. This marker was previously linked to the ASGR in $\mathrm{F}_{1}$ populations developed with $P$. squamulatum and $C$. ciliaris as apomictic pollen parents and validated in a diversity panel of apomictic and sexual Pennisetum and Cenchrus species [13]. The p779/p780 amplicon cosegregated with reproductive mode in a large interspecific $B$. ruziziensis (R. Germ. and C. M. Evrard) $x$ B. decumbens Stapf. $\mathrm{F}_{1}$ mapping population and mapped to a region of reduced recombination on $B$. decumbens chromosome 5 [12]. The psASGR-BBML-specific amplicon was also diagnostic for apomixis in a panel of CIAT genebank accessions from the closely related $B$. brizantha (A. Rich.) Stapf, B. decumbens, B. ruziziensis agamic complex with known reproductive mode [12].

Less is known about the genetic control of apomixis in B. humidicola (Rendle) Schweick (koroniviagrass, syn. Urochloa humidicola (Rendle) Morrone \& Zuloaga). Like the other economically important Brachiaria species ( $B$. brizantha, $B$. decumbens, and B. ruziziensis), B. humidicola, native to East Africa, was introduced to tropical Latin America during the mid-twentieth century [14]. Brachiaria humidicola is highly stoloniferous and well adapted to regions with infertile acidic soil, poor drainage, and seasonal waterlogging [15]. Brachiaria humidicola is estimated to have diverged from the other commercial Brachiaria species in the B. brizantha/B. decumbens/B. ruziziensis agamic complex about 9.46 mya [16]. The distant relationship between $B$. humidicola and the other commercial Brachiaria species is supported by marked differences in inflorescence morphology [14] and a recent phylogenetic study that assessed a large interspecific collection of 261 Brachiaria genotypes with STRUCTURE, neighbor joining (NJ), unweighted pair group method with arithmetic mean (UPGMA), and multiple correspondence analyses [17]. No successful crosses have ever been documented between B. humidicola and any members of the B. brizantha/B. decumbens/B. ruziziensis agamic complex, suggesting that the species are sexually incompatible.

While most Brachiaria species are reported to have a base chromosome number of $\mathrm{x}=9$ [12, 18-22], cytogenetic evidence suggests that the base chromosome number of $B$. humidicola and its close relative $B$. dictyoneura is $\mathrm{x}=6$. Specific evidence for $\mathrm{x}=6$ as the base chromosome number for these species included the presence of hexavalents in accessions with $2 n=36$ and $2 \mathrm{n}=42$ chromosomes and octa- and nonavalents in accessions with $2 \mathrm{n}=54$ chromosomes [23-25]. An allopolyploid origin for $B$. humidicola (AAAABB) was suggested based on meiotic analyses and marker segregation behavior in an simple sequence repeat (SSR)based linkage map developed from a hexaploid $(2 \mathrm{n}=6 \mathrm{x}=$ 36) population derived from a cross of the sexual polyploid B. humidicola accession (CIAT 26146) and the apomictic cultivar 'Tupi' [26].

Brachiaria humidicola exists primarily as a polyploid apomict in nature. Controlled crosses of B. humidicola were first made possible by the discovery of a natural sexual polyploid accession held in the CIAT and EMBRAPA germplasm collections [27]. This sexual polyploid accession, CIAT 26146 (H031 EMBRAPA Beef Cattle) was determined to have 36 chromosomes and used as a female parent in crosses with eighteen apomictic B. humidicola accessions determined to have hexaploid DNA content by flow cytometry [28]. The progeny 
from these crosses formed the basis of the CIAT $B$. humidicola breeding program, which is focused on the development of apomictic cultivars with improved forage quality, productivity, and seed yield.

Only apomictic genotypes can be released as uniform, true-breeding cultivars, while only sexually reproducing genotypes can be used as female parents to develop segregating populations [29]. Because apospory segregates as a single dominant Mendelian factor, each cross between a sexually reproducing female parent and an apomictic pollen donor is expected to produce progeny segregating for reproductive mode on a 1:1 basis. Phenotypic evaluation of reproductive mode in large segregating populations through progeny tests or embryo sac analysis is expensive and time-consuming. Thus, the development of a diagnostic marker test for reproductive mode could shorten breeding cycles and reduce costs [30]. CIAT breeders have used 'N14', a sequence characterized amplified region (SCAR) marker in linkage with the ASGR for routine evaluation of reproductive mode in seedlings from the interspecific $B$. brizantha/B. decumbens/B. ruziziensis breeding program $[30,31]$. Random amplified polymorphic DNA (RAPD) primers producing a band linked to reproductive mode in a $B$. humidicola mapping population have also been reported [32]. Unfortunately, neither 'N14' nor the linked RAPD marker produced polymorphic bands linked to the ASGR in CIAT B. humidicola breeding populations. Fifty-two B. humidicola accessions held in the CIAT genetic resources collection were genotyped with the psASGR-BBML-specific primer pair $\mathrm{p} 779 / \mathrm{p} 780$, and $950 \mathrm{bp}$ amplicons were produced by all accessions except CIAT 26146, the only sexual accession [12]. This finding suggests that p779/p780 might be diagnostic in B. humidicola as well as the agamic complex species. However, p779/p780 has not yet been tested for linkage with the ASGR in segregating $B$. humidicola populations.

Polyploidy, multisomic inheritance, heterozygosity, and self-incompatibility have slowed progress in Brachiaria genomics, but recent advances such as genotyping-by-sequencing (GBS) and bioinformatics pipelines for species lacking reference genomes make the construction of dense maps possible in polyploid apomict species. The primary objective of this study was to develop linkage maps of $B$. humidicola using a hexaploid $(2 \mathrm{n}=6 \mathrm{x}=36) \mathrm{F}_{1}$ mapping population segregating for reproductive mode. These maps were used to assess synteny with the related species foxtail millet (Setaria italica (L.) P. Beauv) and evaluate meiotic interactions among homologous and homeologous linkage groups. The paternal linkage map was also used to locate the position of the ASGR and validate whether p779/p780 is diagnostic for reproductive mode in $B$. humidicola.

\section{Methods}

\section{Materials}

A biparental population of $124 \mathrm{~F}_{1}$ progeny was generated by crossing the sexual accession CIAT 26146 [EM BRAPA Beef Cattle (EBC) H031] to the apomictic male parent CIAT 16888 (EBC H027). The female and male parents of the cross are natural germplasm accessions collected in Burundi and Zimbabwe, respectively. Both parents have been characterized as polyploid accessions with 36 chromosomes [33]. The CIAT 26146 x CIAT 16888 cross was performed by open pollination in the field, where an individual CIAT 26146 plant was surrounded by multiple clonal replicate plants of the apomictic male CIAT 16888. Preliminary analysis of SSR data revealed 12 of 124 progeny to be derived from accidental self-pollination of CIAT 26146. A further 10 progeny were excluded because they had severely distorted GBS results $\left(P<1 \times 10^{-10}\right)$, with an extreme excess of heterozygote calls. The most likely cause of this distortion was determined to be mixing of leaf samples from neighboring plants during tissue collection. Therefore, only 102 total progeny were used for phenotyping, map construction, and subsequent analyses.

\section{Embryo sac analysis}

Inflorescences for embryo sac analysis were harvested from the mapping population progeny in single plant plots at $2 \mathrm{~m}$ spacing at the CIAT research station in Popayán, Colombia (1760 masl; $\left.2.4542^{\circ} \mathrm{N}, 76.6092^{\circ} \mathrm{W}\right)$. Inflorescences were collected in the boot stage and fixed using formalin acetic acid (FAA) for $48 \mathrm{~h}$. Samples were then stored in $70 \% \mathrm{ETOH}$, which was exchanged every $24 \mathrm{~h}$ for three days to remove residual formaldehyde.

The $F_{1}$ progeny of the CIAT 26146 x CIAT 16888 mapping population were classified as apomictic or sexual by cytoembryological observation of methyl salicylate cleared pistils using differential interference contrast (DIC) microscopy [34, 35]. Abnormal (degenerated or ruptured) pistils are common in both apomictic and sexual Brachiaria plants [36]. The number of abnormal pistils was recorded for each of the progeny, and such pistils were excluded from further analyses. A minimum of ten pistils with normally developed embryo sacs were required to assess the reproductive mode of each of the progeny. Progeny with only Polygonum type embryo sac development were classified as sexual, while progeny with any pistils that had enlarged vacuolated nucellar cells or further Panicum type embryo sac development were classified as apomictic. A Chi Squared test was conducted to evaluate whether the population fit the expected segregation ratio for monogenic inheritance of the ASGR. Potential differences in the proportion of abnormal embryo sacs or the number of embryo sacs per pistil in apomictic and sexual progeny were assessed by 
analysis of variance (ANOVA) in SAS 9.2 (SAS Institute Inc., Cary, NC).

\section{Amplified fragment length polymorphism genotyping} Leaf tissue for DNA extractions for amplified fragment length polymorphism (AFLP) and SSR analysis was harvested from parents and progeny grown in greenhouse conditions at CIAT in Palmira, Colombia (1001 masl; $\left.3.5833^{\circ} \mathrm{N}, 76.2500^{\circ} \mathrm{W}\right)$. Genomic DNA for AFLP genotyping was isolated from young leaves following the urea-phenol extraction protocol with slight modifications [37]. Evaluation of progeny with AFLP markers was performed following Vos et al. [38] with slight modifications. Five hundred ng of genomic DNA was digested with Eco RI/Mse I and ligated with Eco RI and Mse I adaptors. Pre-selective amplification of fragments was performed by one-nucleotide extension of $\mathrm{C}$ for the Mse I site and A for the Eco RI site, with a 2 min preliminary extension at $72{ }^{\circ} \mathrm{C}$, followed by 25 cycles of $94{ }^{\circ} \mathrm{C}$ for $20 \mathrm{~s}, 56^{\circ} \mathrm{C}$ for $30 \mathrm{~s}$, and $72{ }^{\circ} \mathrm{C}$ for $2 \mathrm{~min}$, and a $30 \mathrm{~min}$ final extension at $60^{\circ} \mathrm{C}$ in a Gene Amp PCR System 9700 thermocycler (Life Technologies Japan, Tokyo, Japan). Selective amplification was performed with 64 primer combinations of FAM-labeled Eco RI primers and nonlabeled Mse I primers, with three-nucleotide extensions as described in the manufacturer's instructions (Life Technologies Japan, Tokyo, Japan). Selective amplification was performed with a total of 35 cycles plus an initial denaturing step $\left(20 \mathrm{~s}, 94^{\circ} \mathrm{C}\right)$ and a final extension step $\left(2 \mathrm{~min}, 72^{\circ} \mathrm{C}\right)$. The annealing temperature for the first cycle was set at $66^{\circ} \mathrm{C}$, reduced by $1{ }^{\circ} \mathrm{C}$ for each of 10 subsequent cycles, and maintained at $56^{\circ} \mathrm{C}$ for the final 25 cycles, as described by the AFLP Plant Mapping Protocol (Life Technologies). The duration of each annealing step was $30 \mathrm{~s}$. Polymerase chain reactions (PCR) were performed using a Gene Amp PCR System 9700. Amplified DNA fragments were visualized using the ABI-3130 XL (Life Technologies) and GeneMapper v. 5.0 software. Bands that were present in only one of the parental genotypes and fit the 1:1 segregation ratio for presence and absence of bands in the progeny expected for single dose alleles (SDAs) were used in mapping.

\section{Simple sequence repeat marker development and genotyping}

MSATCOMMANDER [39] was used to identify DNA sequences containing SSRs in Roche 454 FLX+ (Roche, Branford, CT) sequence data from the interspecific Brachiaria hybrid cv. Mulato II (CIAT 36087) and B. humidicola cv. Tully (CIAT 679). Primers were developed from SSR-containing sequences using Primer3Plus [40]. The names of SSR primers derived from the DNA sequences of Mulato II and Tully start with "B" and "BC," respectively (Additional file 1: Table $\mathrm{S} 1$ ). These primers were tested for PCR amplification in Mulato II and Tully and the primers producing clear bands were screened for polymorphism in CIAT 26146 and CIAT 16888, the parents of the mapping population. Polymorphic PCR fragments were subsequently tested in the $F_{1}$ mapping population using aliquots from the same DNA extractions used for AFLP genotyping. The SSR marker bands that fit the 1:1 ratio of heterozygotes and homozygotes expected for SDAs were used for mapping. Because only SDA markers were used in map construction, no attempts were made to score allele dosage in the progeny.

Forward primers were designed with a 5'-GGAA ACAGCTATGACCAT $\mathrm{M}-13$ reverse sequence tail for universal fluorescent labeling [41]. Polymerase chain reactions were carried out on a Biometra T1 thermocycler (Analytik Jena AG, Jena, Germany) in $10 \mu \mathrm{L}$ final volume of reaction mixture. The reaction mix consisted of $10 \mathrm{ng}$ genomic DNA, $1.0 \mu \mathrm{M}$ forward and reverse primers, $200 \mu \mathrm{M}$ dNTPs, $0.5 \mu \mathrm{L}$ of AmpliTaq Gold ${ }^{\mathrm{T}}$ DNA polymerase (Applied Biosystems, Foster City, CA), and Buffer II. Initial denaturation was performed at $95^{\circ} \mathrm{C}$ for 7 min. PCR amplifications were then conducted with 11 cycles of $95^{\circ} \mathrm{C}$ for $1 \mathrm{~m}, 65^{\circ} \mathrm{C}$ for $1 \mathrm{~m}$, and $72{ }^{\circ} \mathrm{C}$ for $90 \mathrm{~s}$, followed by 19 cycles of $95^{\circ} \mathrm{C}$ for $1 \mathrm{~m}, 55^{\circ} \mathrm{C}$ for $1 \mathrm{~m}$, and $72{ }^{\circ} \mathrm{C}$ for $90 \mathrm{~s}$, and a final elongation step at $72{ }^{\circ} \mathrm{C}$ for 10 min. Acrylamide gel electrophoresis was performed as described by Yamanaka et al. [42]. Products of PCR were visualized by GelRed ${ }^{\text {sa }}$ (Biotium, Fremont, CA) staining solution and the Pharos FX ${ }^{\mathrm{rm}}$ scanner (Bio-Rad, Hercules, CA) and scored manually.

\section{Single nucleotide polymorphism marker development and genotyping}

We evaluated a $B$. humidicola transcriptome reflecting gene expression under four different physiological stress conditions: high ammonium $(1 \mathrm{mM})$; low nitrogen $(110 \mu \mathrm{M})$ supplied in the form of both ammonium and nitrate; low phosphorus $(1 \mu \mathrm{M})$; and high aluminum $(200 \mu \mathrm{M})$ using adequate amounts of other nutrients in the form of low ionic strength nutrient solution, as described by Wenzl et al. [43]. The root and shoot tissue samples from each parent (CIAT 26146 and CIAT 16888) were collected and subsequently pooled and subjected to Illumina HiSeq2000 sequencing following paired-end library preparation with $500 \mathrm{bp}$ average insert length. The four samples were barcoded and sequenced at Macrogen, obtaining a total of 233 million fragments for the four samples with a read length of $2 \times 100$, for a total production of $47 \mathrm{Gbp}$ of sequence data. Although the read distribution was not completely even and 19 Gbp were assigned to the root tissue of CIAT 16888, at least $8.6 \mathrm{Gbp}$ were obtained for each sample.

Illumina whole genome resequencing (WGS) of one of the progeny of the cross CIAT $26146 \times$ CIAT 16888 was 
also performed. DNA was extracted as described for AFLP genotyping. Paired-end libraries with an average insert length of $500 \mathrm{bp}$ were prepared and sequenced at the Yale Center for Genomic Analysis. This library was sequenced in a full Illumina HiSeq2000 lane. About 200 million fragments were obtained with a read length of $2 \times 76$ bp per fragment. The software SOAPdenovo v2.04 [44] was used to produce a draft assembly using 51 as k-mer size (-K option). The following parameters were set in the soapDeNovo configuration file: asm_flags $=3$, rank $=1$, pair_num_cutoff $=3$ and map_len $=32$. As expected, this produced a very fragmented assembly with 441,785 scaffolds and 2.45 million singleton contigs adding to $1.0 \mathrm{Mbp}$. Because the scaffold N50 was only 1003 $\mathrm{bp}$, most genes were not expected to be assembled in single sequences. Hence, this draft was only used for reference-guided organization of the RNA-seq reads and to extract a DNA context for each single nucleotide polymorphism (SNP) selected to perform kompetitive allele specific PCR (KASP) genotyping. To discriminate single copy and repetitive regions in this assembly, raw WGS reads were aligned to the assembly using bowtie2 v2.2.3 [45] with default parameters except for the maximum number of alignments to retain for each read $(-k)$, which was set to three and the maximum valid insert length $(-\mathrm{X})$ which was set to 1000 . Eighty-five percent of the reads aligned back to the assembly. Alignments were sorted by reference coordinates using Picard (https:// broadinstitute.github.io/picard/). Then, the FindVariants command of the NGSEP pipeline v2.1.5 [46] was used with options -noRD, -noRP and -noSNVs to run only the clustering analysis of reads with multiple alignment to identify repetitive regions.

Because the RNA-seq assay presented above included reads from both CIAT 16888 and CIAT 26146, potential SNPs were identified by aligning the reads of the four samples (root and leave tissues from the two accessions) to the draft genome assembly with bowtie2, and calling variants using the NGSEP pipeline v2.1.5 [46]. The FindVariants command of NGSEP was called with parameters -noRep, -noRD and -noRP to call only SNVs and small indels. All other parameters were left with default values. After merging and genotyping predicted variants within the four RNA-seq samples in an single VCF file, suitable markers for genotyping were selected based on the following criteria: genotyping quality score $(\mathrm{GQ} \geq 40)$ in all samples, only biallelic SNPs, consistent genotyping between tissues of the same individual, location in single copy regions, GC-content 40-65\%, minimum distance to other variants of $40 \mathrm{bp}$, and existence of fewer than 30 unknown bp within a flanking region of $250 \mathrm{bp}$ on either side of the SNP. KASP assays were designed based on the 279 transcriptome-derived SNPs considered suitable for genotyping that were homozygous in the draft
B. humidicola genome assembly and heterozygous in CIAT 16888. This subset of markers was selected for KASP development to increase the chances of identifying SNPs in tight linkage with the ASGR. All KASP assays were used to genotype the full mapping population and parents. Marker reactions were conducted using LGC's genotyping service (LGC Genomics, Beverly, MA) in a $4 \mu \mathrm{L}$ reaction system including $2 \mu \mathrm{L}$ low ROX KASP master mix, $0.106 \mu \mathrm{L}$ of primer mix $(0.318 \mu \mathrm{L}$ of each primer at final concentration) and $2 \mu \mathrm{L}$ of $10-25 \mathrm{ng} / \mu \mathrm{l}$ genomic DNA. The PCR conditions for the KASP assays were $94^{\circ} \mathrm{C}$ for $15 \mathrm{~min}$, followed by 10 cycles of touch down PCR from $68^{\circ} \mathrm{C}$ to $60^{\circ} \mathrm{C}$ with $0.8^{\circ} \mathrm{C}$ decrease per cycle, and 30 cycles of $94^{\circ} \mathrm{C}$ for $20 \mathrm{~s}$ and $57^{\circ} \mathrm{C}$ for $1 \mathrm{~min}$. PCR fluorescent endpoint readings were performed using the Light Cycler 480 Real-Time PCR System (Roche, Germany).

\section{Genotyping with the ASGR-BBML specific primer pair p778/p779}

The parents and progeny of the mapping population were also evaluated with $\mathrm{p} 778 / \mathrm{p} 779$, a primer pair within the Pennisetum squamulatum (L.) R.Br. ASGR-BABY BOOM-like (PSASGR-BBML) candidate gene for the parthenogenesis component of apomixis [13]. Primer sequences and PCR conditions are reported in Worthington et al. [12].

\section{Genotyping by sequencing}

Genotyping by sequencing was performed as described in Worthington et al. [12]. Briefly, libraries were prepared following Heffelfinger et al. [47] with the methylation-sensitive restriction enzyme Hinc II used for digestion. Sequencing was performed as $75 \mathrm{bp}$ pairedend reads on the Illumina HiSeq 2500 in rapid run mode by the Yale Center for Genome Analysis (http://medicine.yale.edu/keck/ycga/index.aspx) following the manufacturer's protocol.

The Tassel 3.0 universal network enabled analysis kit (UNEAK) pipeline was used for de novo SNP discovery and genotype calling [48]. A greater number of reads are required to make accurate genotypic calls in multisomic polyploid populations than diploid populations [49]. Therefore, we first made genotypic calls following recommendations for genotype calling in autotetraploids as described by Li et al. [49]. The threshold for calling a homozygote genotype was set at 11 or more reads of a single allele, while at least two reads of each allele and a minimum minor allele frequency greater than 0.10 was the requirement for calling a heterozygote. If neither condition was met, a missing data score was assigned. No attempt was made to call dosage and distinguish among the multiple genotypes possible for heterozygote individuals. We also performed genotype calling with 
more strict settings, requiring at least 17 reads of a single allele to call a homozygote genotype as recommended for autohexaploids [50]. Maps were constructed separately with each dataset. Because SNP order did not differ significantly between the maps constructed with the two datasets and the maps were more densely saturated with the original tetraploid settings, that data is presented in this manuscript.

The female parent of the mapping population (CIAT 26146) died prior to tissue collection for GBS. Fortunately, we had 12 progeny that were determined to be selfs of CIAT 26146 by SSR and AFLP analyses. These 12 selfed progeny were included in GBS sequencing with CIAT 16888 and the $F_{1}$ progeny from the cross CIAT $26146 \times$ CIAT 16888 and used to impute the female parent genotype for each SNP. CIAT 26146 was called as homozygous for markers that were homozygous in the same allelic state for all 12 selfed progeny. When the selfed progeny were either all heterozygous or segregating for a given SNP, CIAT 26146 was assumed to be heterozygous.

\section{Linkage map construction}

Separate parental linkage maps of CIAT 26146 and CIAT 16888 were created in JoinMap 4.1 following the two-way pseudo-testcross strategy [51]. Markers that were heterozygous in only one parent and had a segregation ratio of less than 2:1 heterozygotes and homozygotes in the 102 progeny were classified as single dose alleles (SDA) and used in mapping. Single dose allele markers that were heterozygous in CIAT 26146 were used to construct the maternal linkage map, while SDAs heterozygous in CIAT 16888 were used to construct the paternal map. Markers with greater than $20 \%$ missing data were excluded from mapping.

Linkage groups were established using an initial threshold linkage logarithm of odds (LOD) score of 7.0. The Monte Carlo maximum likelihood (ML) mapping algorithm with default settings was used to determine marker order and distance within linkage groups. The initial CIAT 16888 linkage map had 38 linkage groups, but two pairs of linkage groups that clustered together at LOD 5.0 and 6.0 respectively were subsequently combined based on shared linkages with double-dose allele (DDA) markers and information on synteny with foxtail millet to form a total of 36 linkage groups. MapChart 2.1 [52] was used to produce charts of the genetic linkage maps.

\section{Synteny analysis and molecular karyotyping}

Single nucleotide polymorphism markers aligned to unique positions in the foxtail millet genome were used to assign linkage groups to chromosomes and identify homologues. To perform the synteny analysis, consensus sequences of SDA tag pairs were extended using partially assembled 30x WGS sequence data from the diploid $B$. ruziziensis accession CIAT 26162 and a B. humidicola progeny from the cross CIAT 26146 x CIAT 16888 (Unpublished data). Tag reads were aligned to the contigs of the partially assembled genomes via NovoAlign (www.novocraft.com). Those contigs were used as the extended tag sequences and queried against the foxtail millet genome (https://phytozome.jgi.doe.gov/pz/portal.html\#!info?alias=Org_Sitalica) [53] using the Basic Local Alignment Search Tool (BLAST) with a cutoff E-value of $<1 \times 10^{-5}$.

Meiotic associations between chromosomal regions with differing degrees of homology and homeology across the maternal and paternal genomes were assessed with high resolution molecular karyotyping [54] as described in Worthington et al. [12]. Each pair of mapped marker alleles was tested for segregation from the expected (1:1:1:1) ratio of individuals with both alleles present $(1 / 1)$, one allele present $(0 / 1$ or $1 / 0)$, and neither allele present $(0 / 0)$ for two alleles at a single homologous locus using Fisher's exact test for count data $(P<0.05)$. Statistical analysis was conducted following Mason et al. [54] with minor modifications and heatmap figures were generated in $\mathrm{R}$ version 3.0.0 (The R Project for Statistical Computing).

Shared linkages with DDA markers were also used to identify homologous linkage groups in the maternal and paternal linkage maps. Single nucleotide polymorphism markers were classified as DDAs based on segregation at a 5:1 ratio of heterozygotes to homozygotes in the $F_{1}$ progeny as expected for markers with tetrasomic inheritance or a 4:1 ratio as expected for markers with hexasomic inheritance according to $X^{2}$ tests $(P>0.05)$ [55]. Linkages between DDA and SDA markers from each parental map were first assessed using the preliminary grouping function of TetraploidMap under simplex-duplex linkages [56]. Because molecular karyotyping suggested that hexasomic inheritance predominated in CIAT 26146, SDA-DDA linkages identified in TetraploidMap for the maternal map were subsequently validated using $X^{2}$ tests $(P>0.05)$ for independence using expected hexasomic genotypic frequencies of independently segregating DDA and SDA markers (Table 1).

\section{Results}

\section{Analysis of reproductive mode}

Eleven of the $102 \mathrm{~F}_{1}$ progeny never flowered over the course of the 18 months that the planting was established in Popayan and therefore could not be assessed for reproductive mode. A further 14 progeny could not be reliably diagnosed as apomictic or sexually reproducing because $74-100 \%$ of the pistils had aborted embryo sacs and it was not possible to reliably evaluate at least 10 pistils with normally developed embryo sacs. The 
Table 1 Expected genotypic ratios for SDA-DDA linkages assuming tetrasomic or hexasomic inheritance ${ }^{a}$

\begin{tabular}{lll}
\hline & Tetrasomic & Hexasomic \\
\hline $\mathrm{AB}^{\mathrm{b}}$ & $1 / 2-1 / 6 \mathrm{r}$ & $1 / 2-1 / 5 \mathrm{r}$ \\
$\mathrm{A}$ & $1 / 6 \mathrm{r}$ & $1 / 5 \mathrm{r}$ \\
$\mathrm{B}$ & $1 / 3+1 / 6 \mathrm{r}$ & $3 / 10+1 / 5 \mathrm{r}$ \\
0 & $1 / 6-1 / 6 \mathrm{r}$ & $1 / 5-1 / 5 \mathrm{r}$ \\
\hline
\end{tabular}

${ }^{a}$ Expected genotypic ratio of progeny for single dose allele (SDA; A000 $\left.\times 0000\right)$ and double dose allele $(\mathrm{DDA} ; \mathrm{BB} 00 \times 0000)$ markers linked in coupling

${ }^{b} A B^{\prime}$ indicates presence of segregating alleles in both SDA and DDA markers, ' $A$ ' indicates presence of the segregating allele for only the SDA marker, ' $B$ ' indicates presence of the segregating allele for only the DDA marker, ' $O$ ' indicates that neither segregating allele is present

remaining $77 \mathrm{~F}_{1}$ progeny which produced 10 or more normally developed embryo sacs segregated for reproductive mode at a $1: 1$ ratio $\left(\chi^{2}=0.117, P=0.73\right)$ (Table 2; Additional file 2: Table S2), as expected for the inheritance of a single dominant genetic factor. The progeny classified as sexually reproducing had only Polygonum type embryo sacs in all normally developed pistils, while the apomictic $F_{1}$ hybrids showed at the minimum one pistil including at least one Panicum type embryo sac. The apomictic progeny had a range of normally developed pistils with Panicum type embryo sacs, Polygonum type embryo sacs, or both. The average proportion of Panicum type embryo sacs

Table 2 Reproductive mode of $77 \mathrm{~F}_{1}$ progeny in the CIAT 26146 x CIAT 16888 mapping population evaluated with embryo sac analysis

\begin{tabular}{lll}
\hline Reproductive mode & Sexual & Apomictic \\
\hline No. of progeny & 37 & 40
\end{tabular}

Proportion of pistils with Panicum-type embryo sacs

$\begin{array}{lll}\text { Mean } & 0 & 0.81 \\ \text { Minimum } & 0 & 0.05 \\ \text { Maximum } & 0 & 1 \\ \text { Standard Deviation } & 0 & 0.21\end{array}$

Proportion of pistils with multiple embryo sacs

$\begin{array}{lll}\text { Mean } & 0 & 0.40 \\ \text { Minimum } & 0 & 0 \\ \text { Maximum } & 0 & 1 \\ \text { Standard Deviation } & 0 & 0.27\end{array}$

Proportion of pistils with abnormal or aborted embryo sacs

\begin{tabular}{lll} 
Mean & 0.41 & 0.13 \\
Minimum & 0 & 0 \\
Maximum & 0.68 & 0.62 \\
Standard Deviation & 0.18 & 0.18 \\
\hline
\end{tabular}

observed in the progeny classified as apomicts was 0.81 and ranged from 0.05-1.00 (Table 2; Additional file 2: Table S2). Only four of the 40 progeny classified as apomicts had $50 \%$ or more Polygonum type embryo sacs. While the sexual progeny exclusively had pistils with single Polygonum type embryo sacs, $40 \%$ of the evaluated pistils in the apomictic progeny had multiple embryo sacs (Table 2; Additional file 2: Table S2). The sexual progeny had significantly more pistils with aborted or abnormal embryo sacs than the apomictic progeny $(P<0.001)$ (Table 2; Additional file 2: Table S2).

\section{Development of GBS and other markers}

The segregating population was used to develop molecular markers and create dense parental linkage maps for CIAT 26146 and CIAT 16888. After quality filtering and processing with the UNEAK pipeline, a total of 51.7 million of the original 499.0 million sequencing reads (Additional file 3: Table S3) were assigned to 208,738 tag pair sites. After markers with over $20 \%$ missing data scores were removed, 6291 polymorphic GBS markers remained. Of these, 3475 markers (55\%) were classified as SDAs, 2288 and 1187 of which were heterozygous in CIAT 26146 and CIAT 16888, respectively. A further $750(12 \%)$ of markers in the dataset fit either a $5: 1$ or $4: 1$ segregation ratio $\left(X^{2}, P<0.05\right)$ and were classified as DDAs. Four hundred and fifty-four of the DDA markers were heterozygous in CIAT 26146, and 296 were heterozygous in CIAT 16888. UNEAK sequences of all mapped GBS-derived markers with variant alleles designated as 'query' and 'hit' according to Lu et al. [48] are given in Additional file 4: Table S4.

A total of 808 AFLP bands were classified as suitable for mapping because they were present in only one of the two parents and fit the 1:1 ratio of presence and absence expected for SDA markers. These bands were generated from 61 primer combinations, which produced between 1 and 47 SDA bands. One hundred and fifty-seven SSR bands produced from 114 primers fit the expected segregation ratio for SDA markers and were used in mapping. Of the 279 transcriptome-derived KASP assays, 160 (57\%) were SDA markers suitable for mapping in this population. Primer sequences for mapped SSRs are given in Additional file 1: Table S1 and KASP primers are listed in Additional file 5: Table S5. A total of 2750 SDA markers were used in the development of the CIAT 26146 maternal haplotype map, including 2288 GBS-derived SNPs, 395 ALFP bands, 67 SSR bands, and 18 KASP markers. A further 1833 SDA markers, including 1187 GBS-derived SNPs, 413 ALFP bands, 90 SSRs, 142 KASP markers, and the ASGR specific marker p779/p780 were assigned to the CIAT 16888 paternal map. 


\section{Genetic linkage maps}

The final CIAT 26146 maternal haplotype map had 2589 markers placed in 36 linkage groups with between 33 and 99 markers per linkage group (Table 3; Fig. 1a; Additional file 6: Table S6). The final map

Table 3 CIAT 26146 and CIAT 16888 parental haplotype maps

\begin{tabular}{|c|c|c|c|c|}
\hline \multirow{2}{*}{$\begin{array}{l}\text { Linkage } \\
\text { Group }\end{array}$} & \multicolumn{2}{|l|}{ CIAT 26146} & \multicolumn{2}{|l|}{ CIAT 16888} \\
\hline & No. of markers & Length (cM) & No. of markers & Length (cM) \\
\hline $1 \mathrm{a}$ & 97 & 86.0 & 94 & 154.1 \\
\hline $1 b$ & 89 & 129.1 & 88 & 151.2 \\
\hline $1 c$ & 82 & 109.8 & 52 & 117.9 \\
\hline $1 d$ & 72 & 92.7 & 46 & 148.4 \\
\hline $1 e$ & 70 & 105.2 & 28 & 88.0 \\
\hline $1 f$ & 65 & 100.4 & 16 & 103.6 \\
\hline $2 \mathrm{a}$ & 93 & 91.8 & 129 & 169.3 \\
\hline $2 b$ & 99 & 97.5 & 59 & 181.0 \\
\hline $2 c$ & 96 & 138.7 & 63 & 126.5 \\
\hline $2 d$ & 87 & 111.9 & 39 & 139.1 \\
\hline $2 e$ & 86 & 106.1 & 17 & 111.0 \\
\hline $2 f$ & 77 & 89.8 & 9 & 68.3 \\
\hline $3 a$ & 72 & 85.1 & 63 & 129.9 \\
\hline $3 b$ & 73 & 68.9 & 52 & 142.3 \\
\hline $3 c$ & 62 & 78.9 & 41 & 139.7 \\
\hline $3 d$ & 58 & 93.9 & 27 & 126.8 \\
\hline $3 e$ & 58 & 77.8 & 12 & 57.4 \\
\hline $3 f$ & 53 & 81.7 & 12 & 79.1 \\
\hline $4 a$ & 93 & 114.2 & 98 & 177.1 \\
\hline $4 b$ & 95 & 108.6 & 71 & 165.3 \\
\hline $4 c$ & 84 & 124.2 & 57 & 155.8 \\
\hline $4 d$ & 81 & 108.6 & 50 & 181.2 \\
\hline $4 e$ & 77 & 101.7 & 20 & 144.8 \\
\hline $4 f$ & 73 & 99.1 & 11 & 85.7 \\
\hline $5 a$ & 82 & 180.0 & 65 & 135.5 \\
\hline $5 b$ & 57 & 108.8 & 57 & 109.9 \\
\hline $5 c$ & 57 & 109.3 & 42 & 132.4 \\
\hline $5 d$ & 46 & 81.4 & 34 & 107.2 \\
\hline $5 e$ & 41 & 60.3 & 29 & 105.8 \\
\hline $5 f$ & 33 & 73.2 & - & - \\
\hline $6 a$ & 82 & 96.2 & 53 & 103.5 \\
\hline $6 b$ & 79 & 96.1 & 48 & 100.0 \\
\hline $6 c$ & 74 & 101.3 & 51 & 123.7 \\
\hline $6 d$ & 59 & 81.1 & 39 & 105.3 \\
\hline $6 e$ & 51 & 58.0 & 17 & 65.0 \\
\hline $6 f$ & 36 & 110.1 & 17 & 63.4 \\
\hline $6 \mathrm{~g}$ & - & - & 15 & 68.0 \\
\hline Total & 2589 & 3557.7 & 1621 & 4362.9 \\
\hline
\end{tabular}

included 2180 GBS SNPs, 332 AFLPs, 61 SSRs, and 16 KASP markers. The total length of the CIAT 26146 haplotype map was $3558 \mathrm{cM}$, with an average marker density of one per $1.37 \mathrm{cM}$. The CIAT 16888 paternal haplotype map consisted of 1621 markers assigned to 36 linkage groups, with nine to 129 markers per linkage group (Table 3; Fig. 1b; Additional file 6: Table S6). The total map length was $4363 \mathrm{cM}$, with an average of one marker per $2.69 \mathrm{cM}$. The final paternal map included 1066 GBS SNPs, 352 AFLPs, 81 SSRs, 121 KASPs, and the indel p779/p780.

\section{Synteny with foxtail millet}

Six hundred and eighty-eight (32\%) of the GBS SNPs and seven (44\%) of the KASP markers heterozygous in CIAT 26146 mapped to unique positions on the foxtail millet reference genome at a cutoff E-value of $<1 \times 10^{-5}$ (Fig. 2a; Additional file 6: Table S6). In the CIAT 16888 parental haplotype map, 356 (33\%) GBS SNPs and 67 (55\%) KASP markers mapped to unique positions on the foxtail millet reference genome (Fig. 2b; Additional file 6: Table S6). The distribution of markers with unique positions on the foxtail millet physical map was uneven across chromosomes, ranging from 187 markers mapped to unique positions on foxtail millet chromosome 9 to just 51 markers mapped to unique positions on chromosome 8 (Fig. 2; Additional file 6: Table S6).

Synteny analysis with foxtail millet indicated that the base chromosome number of $B$. humidicola is $\mathrm{x}=6$. Chromosomes 3, 5, and 6 of B. humidicola were highly collinear with foxtail millet chromosomes 3, 8, and 9, respectively. However, three pairs of foxtail millet chromosomes were fused in B. humidicola. Brachiaria humidicola chromosome 1 consisted of foxtail millet chromosomes 1 and 7, which remained intact and fused together at the proximal tips. Chromosome 2 of $B$. humidicola was composed of foxtail millet chromosome 4 sandwiched in between the two arms of chromosome 2 , with the split on foxtail millet chromosome 2 occurring in the centromeric region between 15.4 and 19.3 Mbp. Likewise, $B$. humidicola chromosome 4 was composed of foxtail millet chromosome 5 split at the centromere between 19.7 and $22.5 \mathrm{Mbp}$, with intact chromosome 6 fused between the two arms (Fig. 2; Additional file 6: Table S6).

\section{Homologous linkage groups and preferential pairing}

Six homologous linkage groups from the CIAT 26146 genetic map corresponding to each of the six base chromosomes of B. humidicola (Fig. 2a; Additional file 6: Table S6) were identified using synteny with foxtail millet and shared linkages with DDA markers. Of the 454 DDA markers that were heterozygous in CIAT 26146, 254 (56\%) were linked in coupling with SDA markers 


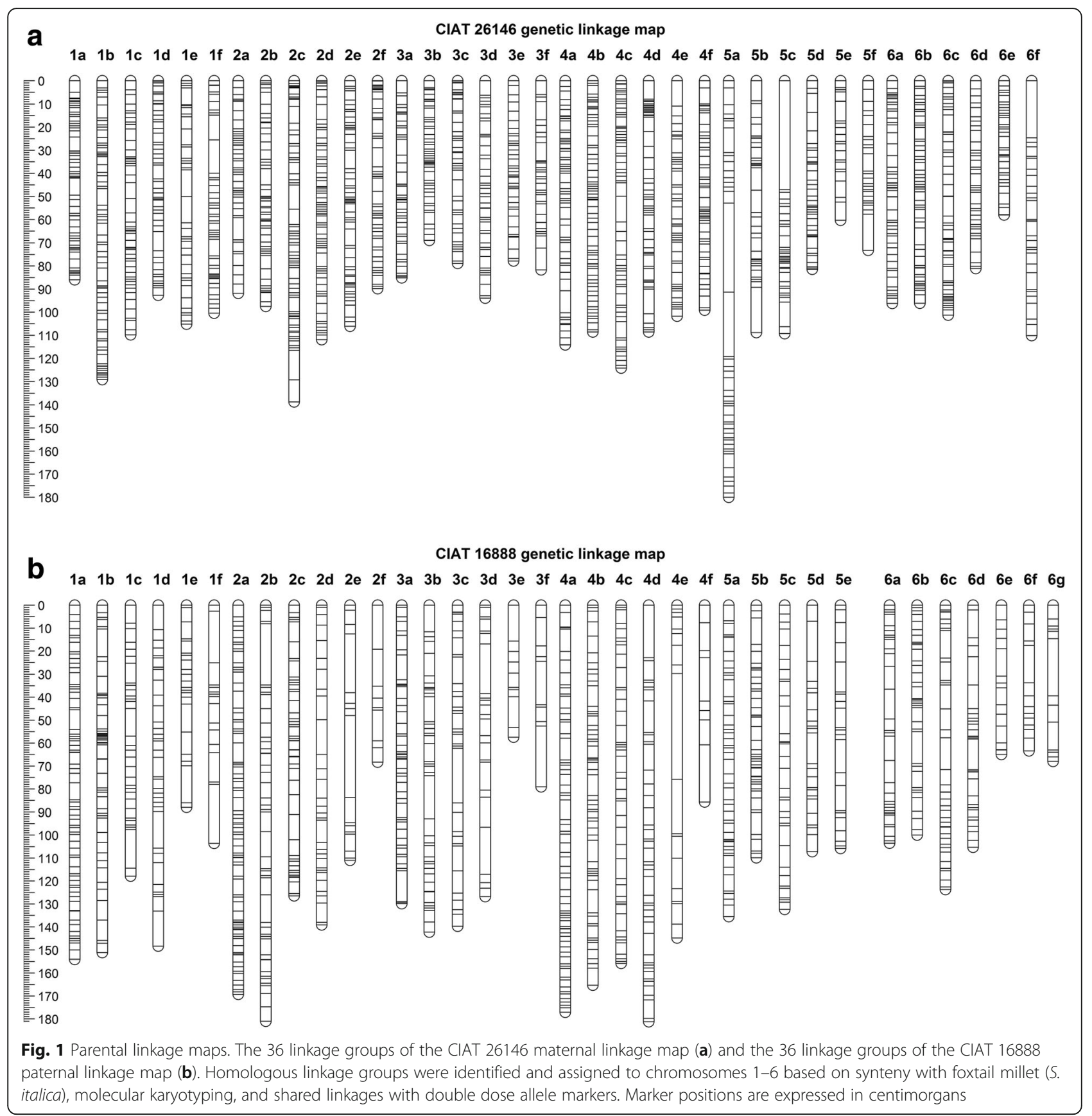

from two homologous linkage groups of the maternal haplotype map (Table 4, Additional file 7: Table S7). The number of DDA markers placed on each chromosome ranged from 14 (chromosome 5) to 63 (chromosome 2). The DDA markers from each base chromosome were linked in coupling with each of the 15 possible pairs of homologs (a-f) at random $\left(X^{2}, P>0.05\right.$; Table 4$)$, suggesting that there was no sub-genome differentiation in CIAT 26146. High-resolution molecular karyotyping also supported random assortment of the six homologous linkage groups of each chromosome in CIAT 26146 (Fig. 3a; Additional file 8: Table S8).

The 36 linkage groups of the CIAT 16888 paternal haplotype map were first assigned to chromosomes based on synteny with foxtail millet and molecular karyotyping results. Synteny analysis showed six linkage groups corresponded to B. humidicola chromosome 1 (a-f), five linkage groups corresponded to chromosomes 2-5 (a-e), and seven linkage groups corresponded to chromosome 6 (a-g) (Fig. 2b; Additional file 6: Table S6). 

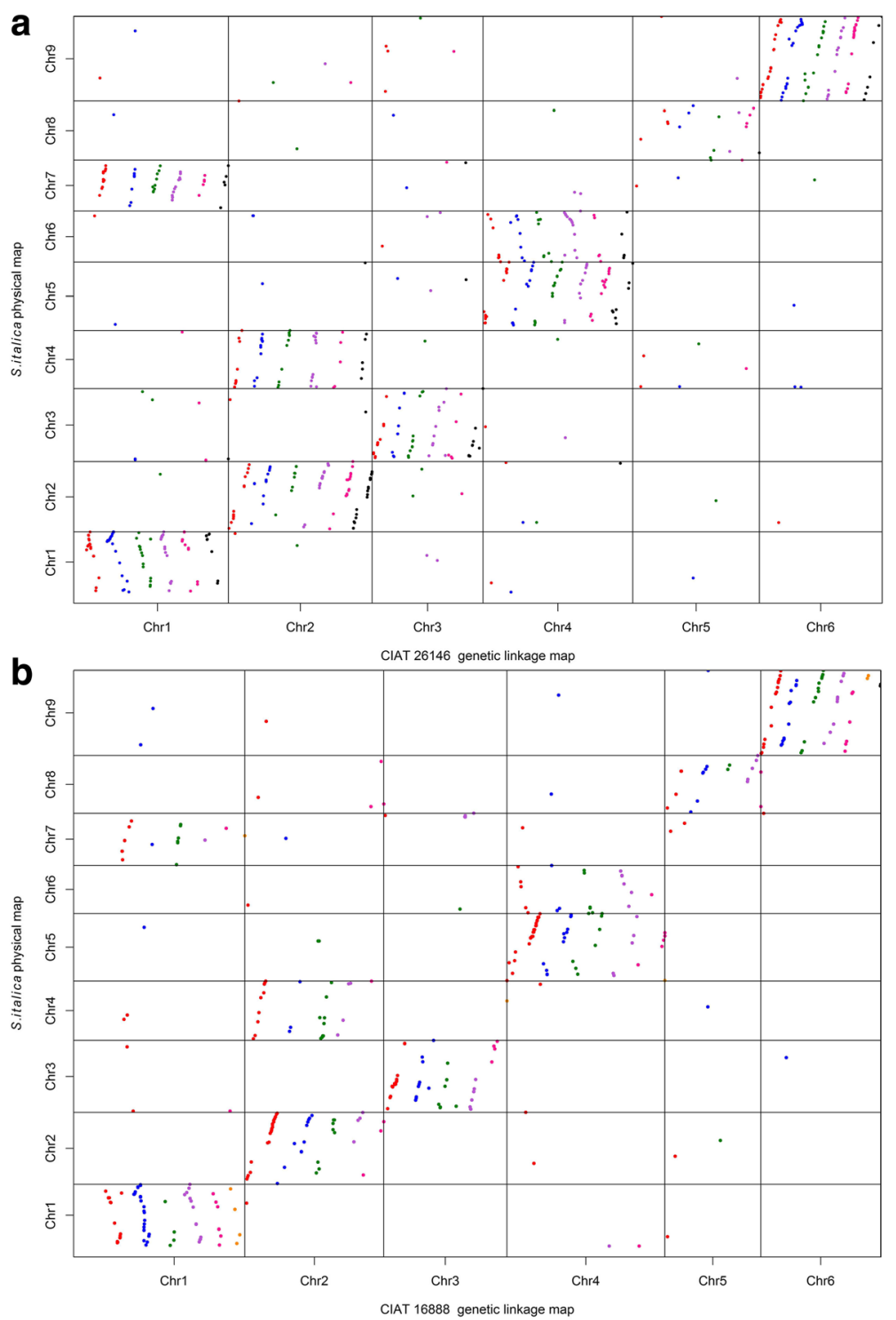

Fig. 2 Synteny with Foxtail Millet. Alignment of markers mapped to the CIAT 26146 (a) and CIAT 16888 (b) genetic linkage maps with unique physical positions on the foxtail millet ( . italica) reference genome at a cutoff of $E$-value $<1 \times 10^{-4}$. Markers mapped to haplotypes a-g of each chromosome are represented with red, blue, green, purple, pink, black, and orange dots

The remaining three linkage groups were assigned to chromosomes 2, 3, and 4 based on segregation patterns revealed in molecular karyotyping analysis (Fig. 3b; Additional file 9: Table S9). Molecular karyotyping indicated that there were two sets of preferentially pairing linkage groups for each chromosome of CIAT 16888 (Fig. 3b; Additional file 9: Table S9). Four homologous linkage groups (a-d) of each chromosome paired at random. On chromosomes $1-4$, the remaining two linkage groups (e-f) paired preferentially with each other, while the fifth homolog (e) of chromosome 5 showed no significant segregation with any other linkage group (Fig. 3c). The three remaining linkage groups (e-g) of chromosome 6 showed significant segregation with each other, though not with homologs (a-d) (Fig. 3d). There was insufficient linkage to combine any of the seven linkage groups of chromosome 6 , even at a linkage LOD of 2.0, suggesting that the unbalanced number of linkage groups assigned to each chromosome may be due to compensated aneuploidy rather than insufficient marker density.

Shared linkages with DDA markers and segregating allele read frequency showed further evidence of sub-genome differentiation in CIAT 16888. Two hundred and nineteen (80\%) of the 296 DDA markers 
Table 4 DDA markers linked in coupling with parental haplotype linkage groups ${ }^{a}$

\begin{tabular}{|c|c|c|c|c|c|c|c|c|c|c|c|c|}
\hline \multirow{2}{*}{$\begin{array}{l}\text { Haplotype } \\
\text { pair }\end{array}$} & \multicolumn{6}{|c|}{ CIAT 26146} & \multicolumn{6}{|c|}{ CIAT 16888} \\
\hline & 1 & 2 & 3 & 4 & 5 & 6 & 1 & 2 & 3 & 4 & 5 & 6 \\
\hline$a, b$ & 5 & 6 & 1 & 6 & 2 & 1 & 6 & 0 & 1 & 2 & 1 & 2 \\
\hline$a, c$ & 2 & 4 & 1 & 1 & 1 & 5 & 2 & 7 & 0 & 6 & 0 & 3 \\
\hline $\mathrm{a}, \mathrm{d}$ & 3 & 2 & 0 & 5 & 2 & 0 & 3 & 9 & 1 & 6 & 1 & 6 \\
\hline$b, c$ & 1 & 1 & 1 & 6 & 0 & 3 & 8 & 15 & 8 & 18 & 5 & 5 \\
\hline$b, d$ & 7 & 3 & 1 & 3 & 1 & 3 & 5 & 6 & 6 & 12 & 2 & 3 \\
\hline$c, d$ & 1 & 2 & 5 & 1 & 1 & 4 & 7 & 8 & 6 & 18 & 2 & 6 \\
\hline$a, e$ & 1 & 1 & 4 & 2 & 2 & 2 & 0 & 0 & 0 & 0 & 0 & 0 \\
\hline$a, f$ & 7 & 4 & 0 & 4 & 0 & 0 & 0 & 0 & 0 & 0 & - & 0 \\
\hline$a, g$ & $-^{c}$ & - & - & - & - & - & - & - & - & - & - & 0 \\
\hline$b, e$ & 3 & 6 & 2 & 4 & 1 & 5 & 0 & 0 & 0 & 0 & 1 & 0 \\
\hline$b, f$ & 3 & 4 & 3 & 0 & 1 & 4 & 0 & 1 & 0 & 0 & - & 0 \\
\hline$b, g$ & - & - & - & - & - & - & - & - & - & - & - & 0 \\
\hline$c, e$ & 4 & 6 & 1 & 4 & 0 & 5 & 0 & 0 & 0 & 0 & 0 & 0 \\
\hline$c, f$ & 1 & 10 & 4 & 6 & 1 & 3 & 0 & 0 & 0 & 0 & - & 0 \\
\hline$c, g$ & - & - & - & - & - & - & - & - & - & - & - & 0 \\
\hline$d, e$ & 2 & 3 & 3 & 6 & 1 & 1 & 0 & 0 & 0 & 0 & 0 & 0 \\
\hline$d, f$ & 1 & 6 & 3 & 1 & 1 & 6 & 0 & 0 & 0 & 0 & - & 0 \\
\hline $\mathrm{d}, \mathrm{g}$ & - & - & - & - & - & - & - & - & - & - & - & 0 \\
\hline$e, f$ & 4 & 5 & 5 & 3 & 0 & 4 & 8 & 0 & 0 & 9 & - & 1 \\
\hline $\mathrm{e}, \mathrm{g}$ & - & - & - & - & - & - & - & - & - & - & - & 0 \\
\hline$f, g$ & - & - & - & - & - & - & - & - & - & - & - & 3 \\
\hline total & 45 & 63 & 34 & 52 & 14 & 46 & 39 & 46 & 22 & 71 & 12 & 29 \\
\hline$p^{b}$ & 0.13 & 0.16 & 0.2 & 0.22 & 0.92 & 0.28 & $<0.001$ & $<0.001$ & $<0.001$ & $<0.001$ & 0.035 & $<0.001$ \\
\hline
\end{tabular}

${ }^{a}$ Number of double dose alleles (DDAs) linked in coupling with each possible pair of SDA haplotypes (a-g) of the six base chromosomes of B. humidicola on the CIAT 26146 and CIAT 16888 genetic maps

${ }^{\mathrm{b}} P$ values for $\mathrm{X}^{2}$ tests of random distribution of shared DDA linkages assuming there is no differentiation of sub-genomes

Indicates that this haplotype pair does not exist for the given chromosome

heterozygous in CIAT 16888 were linked in coupling with two linkage groups corresponding to the same base chromosome from the paternal haplotype map. Between 12 (chromosome 5) and 71 (chromosome 4) DDA markers were in linkage with SDA markers from each chromosome. In contrast to the random distribution of shared DDA linkages among homologs in CIAT 26146, significantly more DDA markers in CIAT 16888 had shared linkages with just four (a-d) homologous linkage groups of each chromosome than would be expected by chance $\left(\chi^{2}, P<0.05\right.$, Table 4; Additional file 7: Table S7). A strong peak in segregating allele read frequency (ratio of reads for the segregating allele to total reads) in GBS SDA markers was observed around 0.25 in the CIAT 16888 haplotype map as expected for an autotetraploid, with lesser peaks at 0.125 and 0.5 (Fig. 4). This finding suggests that while some SNPs were present on all homologs, the majority of SNPs existed in only one of two differentiated sub-genomes of CIAT 16888.

\section{Genetic mapping of the ASGR}

The ASGR was mapped to position $55.8 \mathrm{cM}$ of CIAT 16888 linkage group 1b (Fig. 5; Additional file 6: Table S6). A total of 14 markers, including the ASGR-BBML-specific marker p779/p780, one transcriptome-derived KASP marker, and 12 GBS markers, were in perfect linkage with the ASGR. Although B. humidicola chromosome 1 is a composite of foxtail millet chromosomes 1 and 7, the ASGR mapped to a region that is clearly syntenous with foxtail millet chromosome 1. Six of the GBS markers in perfect linkage with the ASGR mapped to unique positions on foxtail millet chromosome 1 ranging from 3.9 to $26.7 \mathrm{Mbp}$. These markers included TP197082 and TP103482 (3.9 Mbp), TP230498 (10.9 Mbp), TP70501 (12.9 Mbp), TP272804 (23.8 Mbp), and TP207695 (26.7 Mbp). This physically large region includes the centromere of foxtail millet chromosome 1 [53]. The ASGR was flanked at $1.0 \mathrm{cM}$ proximal by the AFLP marker M-CAG/E-AGC140 and at $0.3 \mathrm{cM}$ distal by the GBS marker TP21294. The closest flanking 

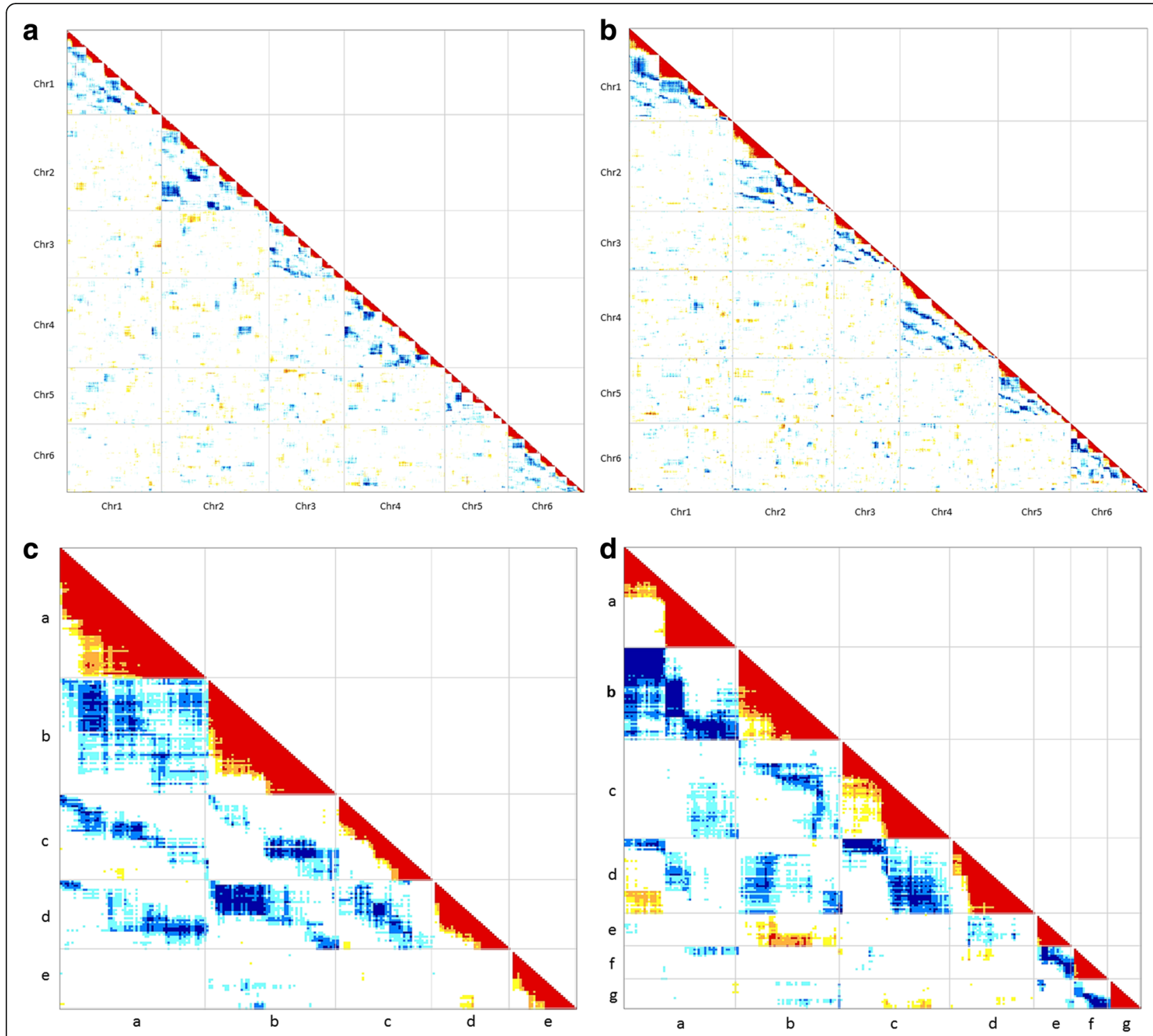

Fig. 3 Molecular karyotyping of the parental linkage maps Linkage and segregation of markers in (a) the CIAT 26146 maternal haplotype map, (b) the CIAT 16888 paternal haplotype map, (c) CIAT 16888 chromosome 5 homologs a-e, and (d) CIAT 16888 chromosome 6 homologs a-g. SNP markers are arranged by their genetic position (not drawn to scale). Genetic regions with statistically significant linkage are indicated in red, orange, and yellow while regions with significant segregation are indicated with shades of blue. Light, medium, and dark blue indicate segregation significant at $0.001<\mathrm{P}$ $<0.05,0.00001<\mathrm{P}<0.001$, and $\mathrm{P}<0.00005$, respectively. Yellow, orange and red indicate linkage significant at $0.001<\mathrm{P}<0.05,0.00001<\mathrm{P}<0.001$, $P<0.00001$, respectively

markers that mapped to unique positions on foxtail millet chromosome 1 were the transcriptome-derived KASP marker scaffold108115 (30.8 Mbp) at $40.30 \mathrm{cM}$ and the GBS marker TP36498 (15.0 Mbp) at $57.7 \mathrm{cM}$.

\section{Discussion}

With over 4210 total mapped markers, the parental maps reported here have over ten times the marker density of the best existing map of B. humidicola [26]. Thirty-six linkage groups were identified for each parental map, as expected based on chromosome counts and flow cytometry $[28,33]$. Dense maps of an interspecific $B$. decumbens $\mathrm{x} B$. ruziziensis mapping population showed that both species had a base chromosome number of $\mathrm{x}=9$ and a high degree of collinearity with foxtail millet, the closest relative of Brachiaria with a publically available reference genome [12]. The only major structural rearrangements discovered between those species and foxtail millet were a reciprocal translocation between the proximal and distal tips of chromosomes 3 and 7 and inversions on chromosomes $1,3,5$, and 6 . In contrast, synteny analysis of the $B$. humidicola parental maps with the 


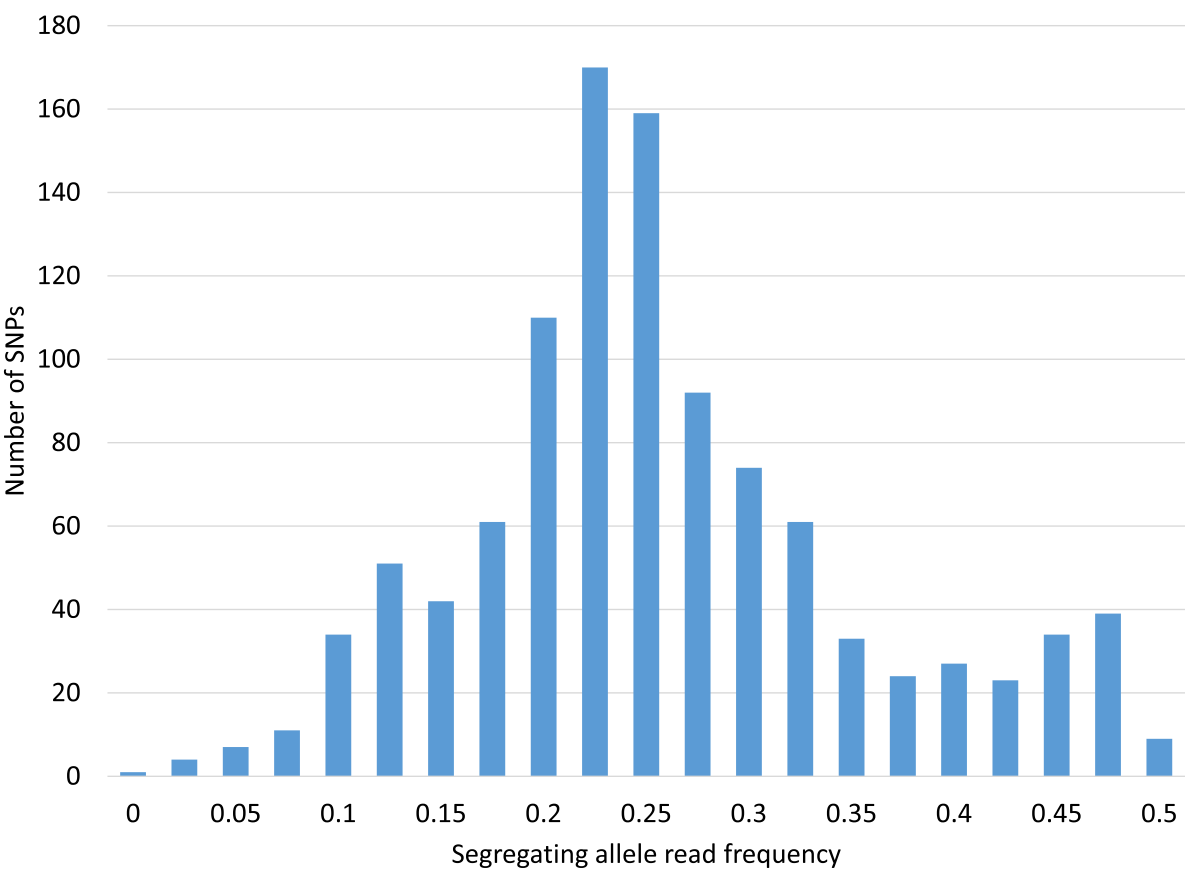

Fig. 4 Read Frequency in CIAT 16888. Relative depth of coverage (read frequency) of the segregating allele in heterozygous loci in the CIAT 16888 paternal linkage map

foxtail millet physical map showed clearly that there were six base chromosomes in this species. This finding is supported by previous cytogenetic evidence for a base chromosome number of $\mathrm{x}=6$ in B. humidicola and B. dictyoneura including the presence of hexavalents and other higher order multivalents in genebank accessions with $2 \mathrm{n}$ $=36$ and higher chromosome numbers $[23,26,57,58]$.

Chromosomes 3, 5, and 6 of B. humidicola had a high degree of synteny with chromosomes 3,8 , and 9 of foxtail millet, B. decumbens, and B. ruziziensis (Fig. 2; Additional file 6: Table S6). The lower base chromosome number of $B$. humidicola relative to $B$. decumbens and B. ruziziensis was accounted for by the fusion of three pairs of chromosomes (S. italica chromosomes 1 and $7=B$. humidicola chromosome $1 ; S$. italica chromosomes 2 and $4=B$. humidicola chromosome 2; and $S$. italica chromosomes 5 and $6=B$. humidicola chromosome 4). This fusion of chromosomes is consistent with the large chromosome size of B. humidicola relative to other Brachiaria species [59]. The strongly conserved synteny between foxtail millet and $B$. decumbens and $B$. ruziziensis suggests that the Brachiaria agamic complex species ( $B$. brizantha, $B$. decumbens, and $B$. ruziziensis) or other $\mathrm{x}=9$ Brachiaria species are likely ancestral to $B$. humidicola.

\section{Meiotic pairing}

Vigna et al. [26] argued for a recent allopolyploid (AABBBB) origin of CIAT 26146 (H031) and the apomictic cultivar 'Tupi' based on the presence of two nucleoli of differing sizes in some meiocytes, the high frequency of disomic and tetrasomic chromosome associations at diakinesis, and differences in sizes of preferentially pairing chromosomes. They suggested that hexaploid $B$. humidicola likely originated from a cross between a sexual diploid female $(2 n=2 x=12$, genome A) and a tetraploid apomictic male $(2 \mathrm{n}=4 \mathrm{x}=24$, genome $B)$ that produced a triploid progeny $(2 n=3 x=18$, $\mathrm{ABB})$ which experienced spontaneous chromosome doubling $(2 n=6 \mathrm{x}=36$, AABBBB $)$. Alternatively, they suggest that the triploid progenitor could have also originated from the cross of two diploid parents $(2 \mathrm{n}=2 \mathrm{x}=$ $12)$, one contributing a reduced gamete $(n=6$, genome A) and the other contributing an unreduced gamete $(n$ $=12$, genome $\mathrm{B}$ ).

Our results from molecular karyotyping, segregating allele read frequency, and DDA-SDA linkage analysis support allopolyploidy (AABBBB) and preferential pairing of sub-genomes in the apomictic male parent CIAT 16888 (Fig. 3b; Fig. 4; Table 4). However, our DDA-SDA linkage results showed no evidence of subgenome differentiation in the sexual female parent (CIAT 26146) (Table 4). Molecular karyotyping analysis also indicated that pairing among the six homologs of each base chromosome in CIAT 26146 occurred at random (Fig. 3a). These findings support hexasomic segregation and possibly autohexaploidy (BBBBBB) in CIAT 26146. 
$1 b$

Si1

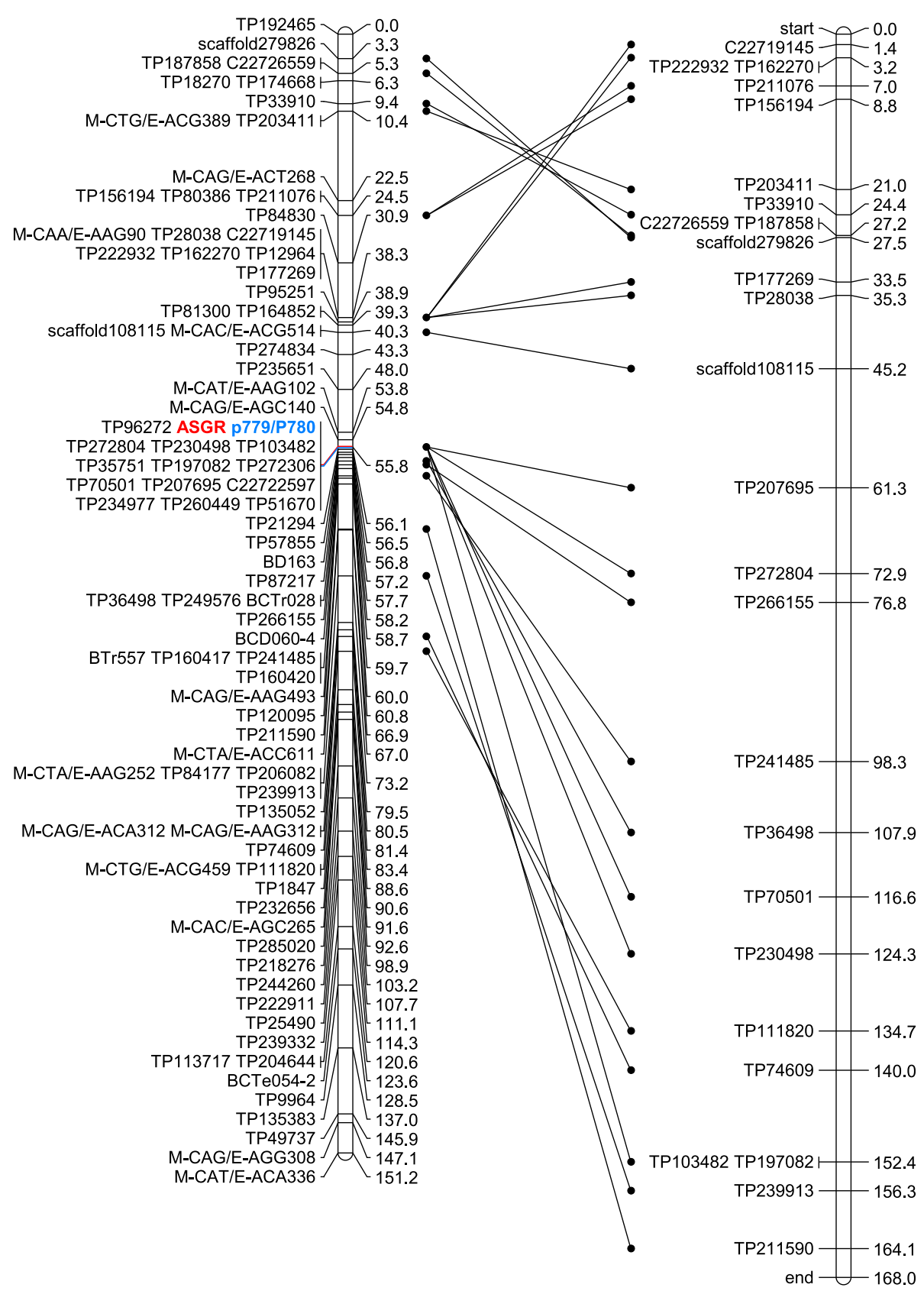

Fig. 5 Comparison of the ASGR carrier chromosome with the physicial map of S. italica chromosome 1. Synteny between CIAT 16888 linkage group $1 \mathrm{~b}$, the carrier chromosome of the apospory specific genomic region (ASGR), and foxtail millet (S. italica) chromosome 1. The ASGR and the ASGR-specific amplicon from p778/p779 are in red and blue bold font respectively. Genetic positions are given in centimorgans. The physical map of foxtail millet chromosome 1 has been inverted to facilitate visualization of synteny and each unit of the physical map represents $2.5 \times 10^{5}$ bp

While it is unexpected that two accessions of the same species would have such different meiotic behavior, two detailed phylogenies of $B$. humidicola have reported that CIAT 26146 is very distantly related to all the apomictic accessions held in the CIAT and EMBRAPA germplasm collections based on STRUCTURE, UPGMA, and NJ analyses [17, 33]. The prevalence of bivalent and tetravalent chromosome associations in diakinesis in CIAT 26146 is not necessarily evidence against autopolyploidy or hexasomic segregation. Bivalents associations predominate in a number of autotetraploid species, including alfalfa (Medicago sativa) [60]. If the sexual and 
apomictic accessions of $B$. humidicola do indeed have different genomic composition (autopolyploid versus allopolyploid), then CIAT 26146 may need to be classified as a separate species or subspecies.

The origin and ancestry of CIAT 26146 remains unknown. Of the $66 \mathrm{~B}$. humidicola accessions maintained in the CIAT Brachiaria collection, CIAT 26146 is the only sexually reproducing genotype. Furthermore, CIAT 26146 is the only documented naturally occurring polyploid sexual genotype in the entire collection of $601 \mathrm{Bra}$ chiaria genotypes maintained by CIAT. Eight other Brachiaria species, including B. bovonei (Chiov.) Robyns, B. brevispicata (Rendle) Stapf, B. dictyoneura (Fig. et De Not.) Stapf, B. jubata (Fig. et De Not.) Stapf, B. platynota (K. Schum.) Robyns, B. reticulata Stapf, B. stigmatisata (Mez) Stapf, and B. subulifolia (Mez) Clayton, were assigned to taxonomic group six with $B$. humidicola based on panicle and inflorescence morphology [14]. Of these species, only $B$. humidicola and $B$. dictyoneura have been used as commercial forages. The noncommercial species of Brachiaria are poorly represented in the CIAT and EMBRAPA germplasm collections relative to commercial species and have been studied less intensively. Comparative genomics and cytological studies including genome in situ hybridization (GISH) with other taxonomic group six species is needed to elucidate the origin, divergence, and evolution of CIAT 26146 and the apomictic $B$. humidicola accessions.

Although 36 linkage groups were found in the apomictic parent CIAT 16888 as expected for a euploid hexaploid genotype $(2 n=6 x=36)$, molecular karyotyping analysis indicated that there were only five linkage groups for chromosome 5 (a-e) and seven linkage groups for chromosome $6(\mathrm{a}-\mathrm{g})$. This evidence for compensated aneuploidy in the apomict CIAT 16888 is not too surprising given that meiotic errors and unbalanced gametes occur with a high degree of frequency in $B$. humidicola $[26,61]$. Furthermore, the high frequency of septaploids $(2 \mathrm{n}=7 \mathrm{x}=42)$ and nonaploids $(2 \mathrm{n}=9 \mathrm{x}=54)$ in the CIAT and EMBRAPA $B$. humidicola collections indicate that this species may be tolerant of aneuploidy [28, 33]. Compensated aneuploidy has been documented in the recently formed natural allopolyploid species Tragopogon miscellus [62] and in experimental neoallopolyploids in the Triticum and Brassica genera [63, 64]. Evidence for segmental allopolyploidy, including frequent non-homologous chromosome pairing, has been documented in B. decumbens [12]. Apomixis has been suggested as a form of meiotic restitution that arrests the process of diploidization and allows polyploid species to remain in a neopolyploid state indefinitely [12]. This evidence of compensated aneuploidy in CIAT 16888 supports the theory that apomixis promotes fertility in meiotically unstable neopolyploid grasses.
The evidence of possible compensated aneuploidy in CIAT 16888 suggests that it may not be a good choice as a male parent in breeding programs. Caryopsis formation rarely exceeds $30 \%$ in Brachiaria [65]. Low seed set is a persistent limitation in Brachiaria, and seed yield potential impacts whether a new variety can be profitably produced and distributed to farmers. Seed production is especially difficult in B. humidicola; seed of $B$. humidicola cultivars 'common' and Llanero was over twice the cost of B. brizantha [66]. Low seed yield in $B$. humidicola may be associated with poor pollen viability. Brachiaria grasses are pseudogamous [3], which means that the secondary nuclei of apomictic embryo-sacs must be fertilized with viable pollen gametes for normal endosperm development and seed production. Abnormal tetrad frequency was correlated with non-viability of pollen grains [67]. An aneuploid apomictic pollen donor is likely to contribute unbalanced gametes to a high frequency of its progeny. While some of these aneuploid progeny may produce acceptable forage biomass, their success as cultivars could be limited by poor seed yield.

\section{Conservation of the ASGR in the Paniceae and translocation to an alternate carrier chromosome}

As expected, the inheritance of apomixis in the CIAT 26146 x CIAT 16888 mapping population fit the segregation ratio for a single dominant Mendelian factor. The ASGR mapped to position $55.8 \mathrm{cM}$ of CIAT 16888 linkage group 1b (Fig. 5; Additional file 6: Table S6), a region syntenic with foxtail millet chromosome 1 . The number of markers in perfect linkage with the ASGR and the large physical distance between them suggests that the ASGR is located in a region of suppressed recombination. Evidence of reduced recombination around the ASGR has also been reported in P. squamulatum [4], $P$. notatum and P. simplex [5-9], and B. decumbens [12].

The location of the ASGR on linkage group $1 \mathrm{~b}$ of $B$. humidicola is surprising given that the ASGR was mapped to position $42.5 \mathrm{cM}$ of $B$. decumbens linkage group $5 \mathrm{c}$, a region syntenous with foxtail millet chromosome 5 [12]. On the other hand, studies in B. brizantha have found that the ASGR was linked to RFLP probes designed from rice chromosome 2 and maize chromosome $5[68,69]$. Both rice chromosome 2 and maize chromosome 5 are mostly syntenic with foxtail millet chromosome 1 [53]. This suggests that B. humidicola may have an ASGR carrier chromosome more closely related to $B$. brizantha than $B$. decumbens. The P. squamulatum ASGR-carrier chromosome was found to be collinear with foxtail millet chromosome 2 and sorghum chromosome 2 by fluorescence in situ hybridization (FISH) and in silico transcript mapping [70].

Three different ASGR-carrier chromosomes (collinear with foxtail millet chromosomes 1, 2, and 5) have been 
identified in the Paniceae to date. The implication of different ASGR-carrier chromosomes has been cited as evidence for independent evolution of apomixis in multiple grass species [71]. However, the perfect linkage of the $A S G R-B B M L$ specific primers $\mathrm{p} 779 / \mathrm{p} 780$ with reproductive mode in independent mapping populations of $B$. decumbens, $B$. humidicola, and $P$. squamulatum indicates that apomixis more likely evolved as a single event and was spread to other species through hybridization or phylogenetic diversification [12, 13]. Comparative genomics with ASGR-linked BACs in Cenchrus and Pennisetum also support the hypothesis of a common origin for aposporous apomixis in the Paniceae tribe $[13,72]$.

\section{Conclusions}

The development of dense molecular maps in hexaploid $B$. humidicola has provided further support for cytogenetic evidence indicating a base chromosome number of six in this species. Analysis of SDA-DDA linkages, synteny with foxtail millet, and molecular karyotyping all supported previous evidence of allopolyploid origin in apomictic B. humidicola. However, these same analyses indicated that there was no significant differentiation of subgenomes or preferential chromosome pairing in the sexually reproducing female parent, CIAT 26146. The evidence of compensated aneuploidy in the apomictic male parent, CIAT 16888, supports the theory that apomixis acts as a form of meiotic restitution that allows unstable polyploid species to remain in a neopolyploid state indefinitely. Our data show that the $A S G R-B B M L$ specific amplicon from p779/p780 was in full linkage with the ASGR in the $F_{1}$ B. humidicola mapping population despite its location on a different carrier chromosome than previously identified in $B$. decumbens. These results provide further evidence of conservation of ASGR-BBML gene sequences across the Paniceae and support their postulation as candidate genes for the parthenogenesis component of apomixis.

\section{Additional files}

Additional file 1: Table S1. Simple sequence repeat (SSR) primers used in the study. (XLSX $18 \mathrm{~kb}$ )

Additional file 2: Table S2. Detailed results of embryo sac analysis. (XLSX $14 \mathrm{~kb}$ )

Additional file 3: Table S3. Depth of genotyping-by-sequencing read coverage in the CIAT 26146 × CIAT 16888 mapping population. (XLSX 13 kb)

Additional file 4: Table S4. UNEAK sequences of GBS derived markers. (XLSX $259 \mathrm{~kb})$

Additional file 5: Table S5. Primer sequences for Kompetitive allele specific PCR (KASP) markers in the CIAT 26146 and CIAT 16888 parental linkage maps. (XLSX $17 \mathrm{~kb})$

Additional file 6: Table S6. Single dose allele marker positions in the CIAT 26146 and CIAT 16888 parental linkage maps, physical positions on the $S$. italica reference genome, deviations from the expected 1:1 ratio of heterozygotes to homozygotes in the $F_{1}$ progeny, and genotype scores in the progeny. (XLSX $1944 \mathrm{~kb}$ )

Additional file 7: Table S7. Double dose allele markers and linkages with SDA haplotypes in the CIAT 26146 and CIAT 16888 parental maps. (XLSX $216 \mathrm{~kb}$ )

Additional file 8: Table S8. Segregation of alleles within the CIAT 26146 genetic map. Marker pairs with statistically significant segregation and co-segregation interactions based on Fisher's exact test for count data are respectively colored orange and blue. (XLSX $50402 \mathrm{~kb}$ )

Additional file 9: Table S9. Segregation of alleles within the CIAT 16888 genetic map. Marker pairs with statistically significant segregation and co-segregation interactions based on Fisher's exact test for count data are respectively colored orange and blue. (XLSX $15261 \mathrm{~kb}$ )

\section{Abbreviations}

AFLP: Amplified fragment length polymorphism; ANOVA: Analysis of variance: ASGR: Apospory-specific genomic region; ASGR-BBML: ASGR-BABY BOOM-like; $B B M$ : $B A B Y$ BOOM; DDA: Double-dose allele; DIC: Differential interference contrast; EBC: EMBRAPA Beef Cattle; GBS: Genotyping-by-sequencing; KASP: Kompetitive allele specific PCR; NJ: Neighbor joining; PCR: Polymerase chain reactions; RAPD: Random amplified polymorphic DNA; SCAR: Sequenced characterized amplified region; SDA: Single-dose allele; SNP: Single nucleotide polymorphism; SSR: Simple sequence repeat; UNEAK: Universal network enabled analysis kit; UPGMA: Unweighted pair group method with arithmetic mean; WGS: Whole genome resequencing

\section{Acknowledgements}

We thank John Miles for designing the cross and developing the mapping population. We also acknowledge CIAT staff whom maintained the population and assisted with the collection of inflorescences for embryo sac analysis including Gerardo Gallego, Aristipo Betancourt, Carlos Dorado, and Gonzalo Rios. We thank Maria Eugenia Recio, Lucia Chavez, and Jonathan Nuñez for their assistance with DNA extraction and JIRCAS staff Midori Hasegawa and Yukie Muraki for their assistance with SSR genotyping. Shin-lchi Tsuruta also made valuable contributions to the design of SSR primers used in this study. Peggy Ozias-Akins and Joanna Conner at the University of Georgia provided invaluable advice and information on primers p779/p780.

\section{Funding}

We acknowledge support from the Yale University High Performance Computing Center (supported by NIH grants RR-19895 and RR-029676-01) and the Yale Center for Genome Analysis. This project has been supported in part by a grant from the National Science Foundation Plant Genome Research Program to SLD (NSF 1444478). Development of SSR markers was supported by the JIRCAS project, "Development of ecologically sustainable agricultural systems through practical use of the biological nitrification inhibition function". Work at CIAT was supported by Federal Ministry for Economic Cooperation and Development (BMZ, Germany) under the GIZ Project Number 11.7860.7-001.00 and the CGIAR Research Program (CRP) on Livestock. We thank all donors that globally support the work of the Livestock CRP through their contributions to the CGIAR system. None of the funding agencies played an active role in the design of the study, its execution, or the writing of the manuscript.

\section{Availability of data and materials}

Sequencing data from this project has been deposited in the NCBI SRA database under bioproject PRJNA509199. Other data generated and analyzed during this study, including primer sequences, UNEAK sequences of GBS derived markers, and genotype scores for the mapping population, are included in this published article and its supplementary information files.

\section{Authors' contributions}

MW, JA, IMR, and JT contributed to overall project conception and design. CQ and YPZ extracted DNA, prepared libraries for genotyping by sequencing, and conducted genotyping with p779/p780. SD and CH coordinated genotyping by sequencing. ME and NY designed primers and conducted AFLP and SSR genotyping. MS and MI coordinated development of transcriptome based KASP markers. CH, JFH, and JD conducted bioinformatics 
analyses. JGP conducted molecular karyotyping analyses. MW conducted linkage mapping, analyzed and interpreted the data, and drafted the manuscript. All authors read and approved the final manuscript.

\section{Ethics approval and consent to participate}

Not applicable.

\section{Consent for publication}

Not applicable.

\section{Competing interests}

The authors declare that they have no competing interests.

\section{Publisher's Note}

Springer Nature remains neutral with regard to jurisdictional claims in published maps and institutional affiliations.

\section{Author details}

IInternational Center for Tropical Agriculture (CIAT), A.A. 6713, Cali, Colombia. ${ }^{2}$ National Agriculture and Food Research Organization (NARO), Institute of Livestock and Grassland Science, Nasushiobara, Tochigi 392-2793, Japan. ${ }^{3}$ Japan International Research Center for Agricultural Sciences (JIRCAS), 1-1 Ohwashi, Tsukuba, Ibaraki 305-8686, Japan. ${ }^{4}$ Department of Molecular, Cellular, and Developmental Biology, Yale University, New Haven, CT 06520, USA. ${ }^{5}$ Present address: Department of Horticulture, University of Arkansas, 306 Plant Sciences Bldg, Fayetteville, AR 72701, USA. ${ }^{6}$ Present address: Systems and Computing Engineering Department, Universidad de los Andes, Bogotá, Colombia. ${ }^{7}$ Present address: Bioinformatics Interdepartmental Ph.D. Program, University of California, Los Angeles, Los Angeles, CA 90095, USA. ${ }^{8}$ Present address: Plant Polymer Research Unit (PPL), National Center for Agricultural Utilization Research (NCAUR), Agricultural Research Service, United States Department of Agriculture (ARS-USDA), 1815 N. University St., Peoria, IL 61604, USA.

\section{Received: 21 January 2018 Accepted: 18 December 2018}

\section{Published online: 14 January 2019}

\section{References}

1. Ozias-Akins P, van Dijk PJ. Mendelian genetics of apomixis in plants. Annu Rev Genet. 2007;41:509-37.

2. Hand ML, Koltunow AMG. The genetic control of apomixis: asexual seed formation. Genetics. 2014;197:441-50.

3. Barcaccia G, Albertini E. Apomixis in plant reproduction: a novel perspective on an old dilemma. Plant Reprod. 2013;26:159-79.

4. Ozias-Akins P, Roche D, Hanna WW. Tight clustering and hemizygosity of apomixis-linked molecular markers in Pennisetum squamulatum implies genetic control of apospory by a divergent locus that may have no allelic form in sexual genotypes. Proc Natl Acad Sci U S A. 1998;95:5127-32.

5. Stein J, Quarin CL, Martínez EJ, Pessino SC, Ortiz JPA. Tetraploid races of Paspalum notatum show polysomic inheritance and preferential chromosome pairing around the apospory-controlling locus. Theor Appl Genet. 2004;109:186-91.

6. Stein J, Pessino SC, Martínez EJ, Rodriguez MP, Siena LA, Quarin CL, et al. A genetic map of tetraploid Paspalum notatum Flügge (bahiagrass) based on single-dose molecular markers. Mol Breed. 2007;20:153-66.

7. Pupilli F, Martinez EJ, Busti A, Calderini O, Quarin CL, Arcioni S. Comparative mapping reveals partial conservation of synteny at the apomixis locus in Paspalum spp. Mol Gen Genomics. 2004;270:539-48.

8. Podio M, Felitti SA, Siena LA, Delgado L, Mancini M, Seijo JG, et al. Characterization and expression analysis of SOMATIC EMBRYOGENESIS RECEPTOR KINASE (SERK) genes in sexual and apomictic Paspalum notatum. Plant Mol Biol. 2014;84:479-95.

9. Calderini O, Chang SB, De Jong H, Busti A, Paolocci F, Arcioni S, et al. Molecular cytogenetics and DNA sequence analysis of an apomixis-linked BAC in Paspalum simplex reveal a non pericentromere location and partial microcolinearity with rice. Theor Appl Genet. 2006;112:1179-91.

10. Conner JA, Mookkan M, Huo H, Chae K, Ozias-Akins P. A parthenogenesis gene of apomict origin elicits embryo formation from unfertilized eggs in a sexual plant. Proc Natl Acad Sci U S A. 2015;112:11205-10 Available from: https://www.pnas.org/content/112/36/11205.long.
11. Boutilier K. Ectopic expression of BABY BOOM triggers a conversion from vegetative to embryonic growth. Plant Cell Online. 2002;14:1737-49.

12. Worthington M, Heffelfinger C, Bernal D, Quintero C, Zapata YP, Perez JG, et al. A parthenogenesis gene candidate and evidence for segmental allopolyploidy in apomictic Brachiaria decumbens. Genetics. 2016;203:1117-32.

13. Akiyama Y, Goel S, Conner JA, Hanna WW, Yamada-Akiyama H, Ozias-Akins P. Evolution of the apomixis transmitting chromosome in Pennisetum. BMC Evol Biol. 2011;11:289 Available from: http://www.biomedcentral.com/14712148/11/289. BioMed Central Ltd.

14. Renvoize SA, Clayton WD, Kabuye CHS. Morphology, taxonomy and natural distribution of Brachiaria (Trin.) Griseb. In: Miles JW, Maass BL, Valle CB, editors. Brachiaria: Biology, Agronomy, and Improvement. Palmira: CIAT; 1996. p. 1-15.

15. Keller-Grein G, Maass BL, Hanson J. Natural variation in Brachiaria and existing germplasm collections. In: Miles JW, Maass BL, Valle CB, editors. Brachiaria: Biology, Agronomy, and Improvement. Palmira: CIAT; 1996. p. 16-35.

16. Pessoa-filho M, Martins AM, Ferreira ME. Molecular dating of phylogenetic divergence between Urochloa species based on complete chloroplast genomes. BMC Genomics. 2017;18:516.

17. Triviño NJ, Perez JG, Recio ME, Ebina M, Yamanaka N, Tsuruta S, et al. Genetic diversity and population structure of Brachiaria species and breeding populations. Crop Sci. 2017;57:2633-44.

18. Valle CB, Savidan YH. Genetics, cytogenetics, and reproductive biology of Brachiaria. In: Miles JW, Maass BL, Valle CB, editors. Brachiaria: Biology, Agronomy, and Improvement. Palmira: CIAT; 1996. p. 147-63.

19. Mendes-Bonato AB, Risso-Pascotto C, Pagliarini MS, Do Valle CB. Chromosome number and meiotic behaviour in Brachiaria jubata (Gramineae). J Genet. 2006;85:83-7.

20. Mendes-Bonato AB, Pagliarini MS, Forli F, Valle CB, Penteado MIO. Chromosome numbers and microsporogenesis in Brachiaria brizantha (Gramineae). Euphytica. 2002;125:419-25.

21. Risso-Pascotto C, Pagliarini MS, Do Valle CB. Microsporogenesis in Brachiaria bovonei (Chiov.) Robyns and B. subulifolia (Mez) Clayton (Poaceae). Sci Agric. 2009;66:691-6.

22. Risso-Pascotto C, Mendes DV, Silva N, Pagliarini MS, Valle CB. Evidence of allopolyploidy in Brachiaria brizantha (Poaceae: Paniceae) through chromosome arrangement at metaphase plate during microsporogenesis. Genet Mol Res. 2006;5:797-803.

23. Boldrini KR, Pagliarini MS, Valle CB. Meiotic behavior of a nonaploid accession endorses $x=6$ for Brachiaria humidicola (Poaceae). Genet Mol Res. 2009;8:1444-50.

24. Boldrini KR, Pagliarini MS, Valle CB. Evidence of natural hybridization in Brachiaria humidicola (Rendle) Schweick. (Poaceae: Panicoideae: Paniceae). J Genet. 2010;89:91-4.

25. Risso-Pascotto C, Pagliarini MS, Do Valle CB. A new basic chromosome number for the genus Brachiaria (Trin.) Griseb. (Poaceae: Panicoideae: Paniceae). Genet Resour Crop Evol. 2006;53:7-10.

26. Vigna BBZ, Santos JCS, Jungmann L, do Valle CB, Mollinari M, Pastina MM, et al. Evidence of allopolyploidy in Urochloa humidicola based on cytological analysis and genetic linkage mapping. PLoS One. 2016;11:e0153764. Available from: https://doi.org/10.1371/journal.pone.0153764.

27. Valle $\mathrm{CB}$, Glienke C. New sexual accessions in Brachiaria. Apomixis News Let. 1991;3:11-3.

28. Penteado MIDO, dos Santos ACM, Rodrigues IF, Valle CB, Seixas MAC, Esteves A. Determinacao de ploidia e avaliacao da quantidade de DNA total em diferentes especies do genero Brachiaria. EMBRAPA Gado de Corte: Campo Grande; 2000.

29. Miles JW, Cardona C, Sotelo G. Recurrent selection in a synthetic brachiariagrass population improves resistance to three spittlebug species. Crop Sci. 2006:46:1088-93.

30. Worthington ML, Miles JW. Reciprocal Full-sib Recurrent Selection and Tools for Accelerating Genetic Gain in Apomictic Brachiaria. In: Budak H, Spangenberg G, editors. Molecular Breeding of Forage and Turf. Cham: Springer; 2015. p. 19-30.

31. Pedraza Garcia FP. Hacia la localización del gen de apomixis en Brachiaria usando marcadores moleculares RAPD [thesis]. Palmira: Universidad Nacional de Colombia; 1995.

32. Zorzatto C, Chiari L, Araújo Bitencourt G, Valle CB, Campos Leguizamón GO, Schuster I, et al. Identification of a molecular marker linked to apomixis in Brachiaria humidicola (Poaceae). Plant Breed. 2010;129:734-6.

33. Jungmann $L$, Vigna $B B Z$, Boldrini $K R$, Sousa $A C B$, Valle $C B$, Resende $R M S$, et al. Genetic diversity and population structure analysis of the tropical 
pasture grass Brachiaria humidicola based on microsatellites, cytogenetics, morphological traits, and geographical origin. Genome. 2010;53:698-709.

34. Young BA, Sherwood RT, Bashaw EC. Cleared-pistil and thick-sectioning techniques for detecting aposporous apomixis in grasses. Can J Bot. 1979; 57:1668-72.

35. Nakagawa $\mathrm{H}$. Embryo sac analysis and crossing procedure for breeding apomictic guineagrass (Panicum maximum Jacq.). Japan Agric Res Q. 1990; 24:163-8.

36. Valle CB, Savidan YH, Jank L. Apomixis and sexuality in Brachiaria decumbens Stapf. Nice: Proceedings of the XVI International Grasslands Congress; 1989. p. 407-8.

37. Shure M, Wessler S, Fedoroff N. Molecular identification and isolation of the waxy locus in maize. Cell. 1983;35:225-33.

38. Vos $P$, Hogers R, Bleeker M, Reijans M, van de Lee T. A new technique for DNA fingerprinting. Nucleic Acids Res. 1995;44:388-96.

39. Faircloth BC. MSATCOMMANDER: detection of microsatellite repeat arrays and automated, locus-specific primer design. Mol Ecol Resour. 2008;8:92-4

40. Untergasser A, Nijveen $H$, Rao X, Bisseling T, Geurts R, Leunissen JAM Primer3Plus, an enhanced web interface to Primer3. Nucleic Acids Res. 2007;35:71-4.

41. Schuelke M. An economic method for the fluorescent labeling of PCR fragments. Nat Biotech. 2000;18:233-4.

42. Yamanaka N, Hasran M, Xu DH, Tsunematsu H, Idris S, Ban T. Genetic relationship and diversity of four Mangifera species revealed through AFLP analysis. Genet Resour Crop Evol. 2006;53:949-54.

43. Wenzl P, Mancilla LI, Mayer JE, Albert R, Rao IM. Simulating infertile acid soils with nutrient solutions. Soil Sci Soc Am J. 2003;67:1457-69.

44. Luo R, Liu B, Xie Y, Li Z, Huang W, Yuan J, et al. SOAPdenovo2: an empirically improved memory-efficient sort read de novo assembler Gigascience. 2012;1:18.

45. Langmead B, Salzberg SL. Fast gapped-read alignment with bowtie 2. Nat Methods. 2012;9:357-9.

46. Duitama J, Quintero JC, Cruz DF, Quintero C, Hubmann G, Foulquié-Moreno $M R$, et al. An integrated framework for discovery and genotyping of genomic variants from high-throughput sequencing experiments. Nucleic Acids Res. 2014;42:e44

47. Heffelfinger C, Fragoso CA, Moreno MA, Overton JD, Mottinger JP, Zhao H et al. Flexible and scalable genotyping-by-sequencing strategies for population studies. BMC Genomics. 2014;15:979.

48. Lu F, Lipka AE, Glaubitz J, Elshire R, Cherney JH, Casler MD, et al. Switchgrass genomic diversity, ploidy, and evolution: novel insights from a networkbased SNP discovery protocol. PLoS Genet. 2013;9:e1003215.

49. Li X, Wei Y, Acharya A, Jiang Q, Kang J, Brummer EC. A saturated genetic linkage map of autotetraploid alfalfa (Medicago sativa L.) developed using genotyping-by-sequencing is highly syntenous with the Medicago truncatula genome. G3. 2014;4:1971-9.

50. Melo ATO, Bartaula R, Hale I. GBS-SNP-CROP: a reference-optional pipeline for SNP discovery and plant germplasm characterization using variable length, paired-end genotyping-by-sequencing data. BMC Bioinformatics. 2016;17:29.

51. Van Ooijen JW. Multipoint maximum likelihood mapping in a full-sib family of an outbreeding species. Genet Res. 2011;93:343-9.

52. Voorrips RE. MapChart: software for the graphical presentation of linkage maps and QTLs. J Hered. 2002;93:77-8.

53. Zhang G, Liu X, Quan Z, Cheng S, Xu X, Pan S, et al. Genome sequence of foxtail millet (Setaria italica) provides insights into grass evolution and biofuel potential. Nat Biotechnol. 2012;30:549-54.

54. Mason AS, Batley J, Bayer PE, Hayward A, Cowling WA, Nelson MN. Highresolution molecular karyotyping uncovers pairing between ancestrally related Brassica chromosomes. New Phytol. 2014:202:964-74.

55. Kriegner A, Cervantes JC, Burg K, Mwanga ROM, Zhang DP. A genetic linkage map of sweetpotato Ipomoea batatas (L.) Lam. based on AFLP markers. Mol Breed. 2003;11:169-85.

56. Hackett CA, Milne I, Bradshaw JE, Luo Z. TetraploidMap for windows: linkage map construction and QTL mapping in autotetraploid species. J Hered. 2007;98:727-9.

57. Risso-Pascotto C, Pagliarini MS, do Valle CB. Microsporogenesis in Brachiaria dictyoneura (Fig. \& De not.) Stapf (Poaceae: Paniceae). Genet Mol Res. 2006; 5:837-45.
58. Boldrini KR, Adamowski EV, Silva N, Pagliarini MS, Valle CB. Meiotic behavior in nonaploid accessions of Brachiaria humidicola (Poaceae) and implications for breeding. Genet Mol Res. 2011;10:169-76.

59. Bernini C, Marin-Morales MA. Karyotype analysis in Brachiaria (Poaceae) species. Cytobios. 2001;104:157-71.

60. Stanford EH, Clement WM. Cytology and crossing behavior of a haploid alfalfa plant. Agron J. 1958;50:598-92.

61. Boldrini KR, de Adamowski EV, Message H, Calisto V, Pagliarini MS, Valle CB. Meiotic behavior as a selection tool in the breeding of Brachiaria humidicola (Poaceae). Euphytica. 2011;182:317-24.

62. Chester M, Gallagher JP, Symonds W, Cruz da Silva AV, Mavrodiev EV, Leitch $A R$, et al. Extensive chromosomal variation in a recently formed natural allopolyploid species, Tragopogon miscellus (Asteraceae). Proc Natl Acad Sci. 2012;109:1176-81.

63. Xiong Z, Gaeta RT, Pires JC. Homoeologous shuffling and chromosome compensation maintain genome balance in resynthesized allopolyploid Brassica napus. Proc Natl Acad Sci. 2011;108:7908-13.

64. Mestiri I, Chagué V, Tanguy A, Huneau C, Huteau V, Belcram H, et al. Newly synthesized wheat allohexaploids display progenitor-dependent meiotic stability and aneuploidy but structural genomic additivity. New Phytol. 2010; 186:86-101.

65. Hopkinson J, Souza F, Diulgheroff S, Ortiz A, Sanchez M. Reproductive physiology, seed production, and seed quality of Brachiaria. In: Miles JW, Maass BL, Valle CB, editors. Brachiaria: Biology, Agronomy, and Improvement. Palmira: CIAT; 1996. p. 125-40.

66. Jank L, Barrios SC, Valle CB, Simeao RM, Alves GF. The value of improved pastures to Brazilian beef production. Crop Pasture Sci. 2014;65:1132-7.

67. Souza VF, Pagliarini MS, Valle CB, Bione NCP, Menon MU. Meiotic behavior of Brachiaria decumbens hybrids. Genet Mol Res. 2015;14:12855-65.

68. Pessino SC, Evans C, Ortiz JPA, Armstead I, Valle CB, Hayward MD. A genetic map of the apospory-region in Brachiaria hybrids: Identification of two markers closely associated with the trait. Hereditas. 1998;128:153-8.

69. Pessino SC, Ortiz JPA, Leblanc O, Valle CB, Evans C, Hayward MD. Identification of a maize linkage group related to apomixis in Brachiaria. Theor Appl Genet. 1997;94:439-44.

70. Sapkota S, Conner JA, Hanna WW, Simon B, Fengler K, Deschamps S, et al. In silico and fluorescence in situ hybridization mapping revealscollinearity between the Pennisetum squamulatum apomixis carrier-chromosome and chromosome 2 of sorghum and foxtail millet. PLoS One. 2016;11:e0152411.

71. Jessup RW, Burson BL, Burow GB, Wang YW, Chang C, Lia Z, et al. Disomic inheritance, suppressed recombination, and allelic interactions govern apospory in buffelgrass as revealed by genome mapping. Crop Sci. 2002;42:1688-94.

72. Ozias-Akins P, Akiyama Y, Hanna WW. Molecular characterization of the genomic region linked with apomixis in Pennisetum/Cenchrus. Funct Integr Genomics. 2003:3:94-104.

Ready to submit your research? Choose BMC and benefit from

- fast, convenient online submission

- thorough peer review by experienced researchers in your field

- rapid publication on acceptance

- support for research data, including large and complex data types

- gold Open Access which fosters wider collaboration and increased citations

- maximum visibility for your research: over $100 \mathrm{M}$ website views per year

At $\mathrm{BMC}$, research is always in progress.

Learn more biomedcentral.com/submissions 